International Journal of Bifurcation and Chaos, Vol. 11, No. 4 (2001) 1121-1140

(c) World Scientific Publishing Company

\title{
SELF-OSCILLATIONS AND SLIDING IN RELAY FEEDBACK SYSTEMS: SYMMETRY AND BIFURCATIONS
}

\author{
MARIO DI BERNARDO* \\ Department of Engineering Mathematics, \\ University of Bristol, Bristol BS8 1TR, UK \\ KARL HENRIK JOHANSSON ${ }^{\dagger}$ \\ Department of Signals, Sensors and Systems, \\ Royal Institute of Technology, 10044 Stockholm, Sweden \\ FRANCESCO VASCA ${ }^{\ddagger}$ \\ Facoltá di Ingegneria, Universitá del Sannio in Benevento, \\ Corso Garibaldi 107, 82100 Benevento, Italy
}

Received October 22, 1999; Revised June 20, 2000

\begin{abstract}
This paper is concerned with the bifurcation analysis of linear dynamical systems with relay feedback. The emphasis is on the bifurcations of the system periodic solutions and their symmetry. It is shown that, despite what has been conjectured in the literature, a symmetric and unforced relay feedback system can exhibit asymmetric periodic solutions. Moreover, the occurrence of periodic solutions characterized by one or more sections lying within the system discontinuity set is outlined. The mechanisms underlying their formation are carefully studied and shown to be due to an interesting, novel class of local bifurcations.
\end{abstract}

\section{Introduction}

Relay feedback is one of the most commonly used control techniques in practical applications. The idea of using a switching action to achieve heat control was studied already in 1887 by Hawkins, who showed that a temperature control system tends to oscillate under such discontinuous control [Hawkins, 1887]. Since then many engineering applications of relay feedback have been presented in the literature. Early examples come from mechanical and electromechanical systems [Flügge-Lotz, 1953; Andronov, et al., 1965; Tsypkin, 1984], while recent attention has been motivated by variable-structure controllers [Utkin, 1992], supervisory switched control [Morse, 1995], relay methods for tuning controllers in pro- cess industry [Åström \& Hägglund, 1995], hybrid systems [Brockett, 1993] and delta-sigma converters in signal processing [Norsworthy et al., 1997].

Various mathematical methods have been proposed to investigate the dynamics of relay feedback systems. These systems tend to oscillate also without external excitation, and particular interest has been devoted to detect such self-oscillations; for example, by using describing functions and other frequency methods [Tsypkin, 1984; Atherton 1975]. Although relay systems have been studied for more than a century, several problems remain unsolved. For instance, the global stability of periodic solutions is largely an open question [Åström, 1995; Megretski, 1996; Johansson et al., 1999; Gonçalves et al., 1999].

\footnotetext{
*Author for correspondence.

E-mail: m.dibernardo@bristol.ac.uk

${ }^{\dagger}$ E-mail: kallej@s3.kth.se

${ }^{\ddagger}$ E-mail: vasca@unisannio.it
} 
It has been shown that even low-order relay feedback systems can exhibit a number of quite complicated self-oscillations. The existence of periodic solutions with sliding, here called sliding orbits, has been reported in [Johansson et al., 1999] and independently in [Neimark, 1972; Fedosenko, 1976], where sliding refers to an infinite number of relay switchings [Utkin, 1992; Filippov, 1988; Aizerman \& Pyatnitskii, 1974]. The existence of a similar type of periodic solution, but with an arbitrarily large finite number of relay switchings per period, is shown in [Johansson et al., 1997; di Bernardo et al., 1998]. Quasiperiodic solutions in relay feedback systems with hysteresis as well as chaotic attractors have also been detected and are discussed in [Cook, 1985; Amrani \& Atherthon, 1989; Genesio \& Tesi, 1990]. In [Holzhueter \& Klinker, 1998], it has been proposed that, under certain conditions, these chaotic regimes can be explicitly used in the control action.

The main aim of this paper is to study the bifurcation scenarios leading to the formation of asymmetric periodic solutions and sliding orbits in relay feedback systems. We show that a symmetric periodic solution can give rise to a pair of asymmetric orbits through a symmetry-breaking bifurcation. Thus, we present a counterexample to the commonly stated conjecture that asymmetric periodic solutions cannot exist for relay feedback systems, unless either an external forcing term is added to the system or the relay device is made asymmetric [Tsypkin, 1984]. Moreover, we uncover the bifurcation mechanism, termed sliding bifurcation, through which sliding motion can become an integral part of a periodic solution for a relay feedback system. Further analysis leads to the discovery of a whole new class of bifurcations to more complex solutions such as the so-called multisliding orbits, characterized by more than one sliding section. These bifurcations will be also related to those commonly observed in general piecewise smooth dynamical systems, such as the so-called border-collision and grazing bifurcations [Nusse \& Yorke, 1994; Feigin, 1970, 1974; Nordmark, 1991] which have been detected in several systems of relevance in applications [Dankowicz \& Nordmark, 2001; di Bernardo et al., 1998a; di Bernardo et al., 1998b]. Numerical evidence of possible chaotic regimes will also be presented.

The outline of the paper is as follows. Section 2 introduces the equations describing an $n$ dimensional relay feedback system and gives a brief discussion on the occurrence of sliding motions. A third-order relay feedback example, which will serve as an illustrative example throughout the paper, is then presented. Section 3 is devoted to the analysis of periodic solutions in relay feedback systems. An appropriate set of Poincaré maps is introduced to study their existence and stability and is then used to perform the subsequent bifurcation analysis. In Sec. 4, the symmetry of the system orbits is discussed. It is shown that asymmetric orbits can indeed occur in the systems under investigation and are generated through a symmetry-breaking bifurcation of symmetric periodic solutions. The occurrence of limit cycles with sliding is discussed in Sec. 5 and the bifurcation underlying the transition from nonsliding to sliding orbits is presented in Sec. 6. The bifurcations of these sliding solutions is then discussed in Sec. 7 where the existence of orbits characterized by multiple sliding segments is outlined. It is suggested that these multisliding orbits are generated through a peculiar bifurcation scenario. Evidence of seemingly aperiodic attractors is finally given in Sec. 8 together with the conclusions and suggestions for further work.

\section{Relay Feedback System}

The class of relay feedback systems that we consider consists of a single-input single-output linear time-invariant (LTI) system for which the control variable is obtained via a unit negative feedback of the output variable. Thus, the relay feedback system under investigation has the state-space representation:

$$
\begin{aligned}
& \dot{x}=A x+B u, \\
& y=C x, \\
& u=-\operatorname{sgn} y,
\end{aligned}
$$

where $A \in \mathcal{R}^{n \times n}, B \in \mathcal{R}^{n \times 1}$ and $C \in \mathcal{R}^{1 \times n}$ are constant matrices. The input $u$ and output $y$ of the linear part of the system are scalar functions, while $x=\left(x^{(1)}, x^{(2)}, \ldots, x^{(n)}\right)$ has $n \geq 1$ components. The sign function is defined as $\operatorname{sgn} y=1$ if $y>0, \operatorname{sgn} y=-1$ if $y<0$, and $\operatorname{sgn} y \in[-1,1]$ is a set-valued mapping.

A solution $x:[0, \infty) \mapsto \mathcal{R}^{n}$ of (1)-(3) is interpreted in the sense of [Filippov, 1988], i.e. as any absolutely continuous function that satisfies the system equations. Note there may be nonunique solutions. We say that a solution $x^{*}:[0, \infty) \mapsto \mathcal{R}^{n}$ of (1)-(3) is periodic, if there exists $T>0$ such that $x^{*}(t+T)=x^{*}(t)$ for all $t>0$. 
If not stated otherwise, the so-called observer canonical form is assumed, i.e. the system matrices in (1) and (2) are assumed to have the following structure:

$$
\begin{gathered}
A=\left(\begin{array}{ccccc}
-a_{1} & 1 & 0 & \ldots & 0 \\
-a_{2} & 0 & 1 & & 0 \\
\vdots & & & \ddots & \vdots \\
-a_{n-1} & 0 & 0 & & 1 \\
-a_{n} & 0 & 0 & \ldots & 0
\end{array}\right), \quad B=\left(\begin{array}{c}
b_{1} \\
b_{2} \\
\vdots \\
b_{n-1} \\
b_{n}
\end{array}\right) \\
C=\left(\begin{array}{c}
1 \\
0 \\
\vdots \\
0 \\
0
\end{array}\right)^{T},
\end{gathered}
$$

which corresponds to the transfer function

$$
\begin{aligned}
G(s) & =C(s I-A)^{-1} B \\
& =\frac{b_{1} s^{n-1}+b_{2} s^{n-2}+\cdots+b_{n-1} s+b_{n}}{s^{n}+a_{1} s^{n-1}+a_{2} s^{n-2}+\cdots+a_{n-1} s+a_{n}} .
\end{aligned}
$$

For simplicity, we assume throughout the paper that $a_{n} \neq 0$ so that $A$ is nonsingular.

The system (1)-(3) switches from one LTI configuration to the other whenever the output $y$ changes its sign, i.e. whenever the state $x$ crosses the switching hyperplane defined as:

$$
S=\left\{x \in \mathcal{R}^{n}: C x=0\right\} .
$$

Note that for every initial condition outside $S$, the state trajectory will eventually cross $S$ if the steady-state gain $G(0)$ is positive and $G$ is stable [Johansson et al., 1999].

Finally, note that relay feedback systems such as (1)-(3) are instances of a wider class of piecewise smooth dynamical systems of the form:

$$
\dot{x}=F(x)= \begin{cases}f_{1}(x), & \sigma(x)<0 \\ f_{2}(x), & \sigma(x)>0\end{cases}
$$

with $x \in \mathcal{R}^{n}, f_{1}, f_{2}: \mathcal{R}^{n} \mapsto \mathcal{R}^{n}$ smooth vector functions and $\sigma$ being an odd function of $x$.

These systems are said to be symmetric if

$$
\begin{aligned}
& f_{1}(x)=-f_{2}(-x), \\
& f_{2}(x)=-f_{1}(-x) .
\end{aligned}
$$

In fact, if this is true, we have

$$
F(x)=-F(-x)
$$

similarly to what is usually assumed for $Z_{2}$ symmetric smooth dynamical systems [Swift \& Wiesenfeld, 1984]. Thus, system (1)-(3) is also symmetric.

It is worth mentioning here that (6) is used to model a wide variety of other engineering systems including DC/DC power converter [di Bernardo et al., 1998a, 1998b], friction oscillators [Dankowics \& Nordmark, 2001; Popp \& Shelter, 1990] and many other devices.

\subsection{Sliding motion}

Under certain conditions, it has been shown that relay feedback systems can exhibit solutions lying within the system discontinuity set $S$ [Johansson et al., 1999; Neimark, 1972]. This peculiar type of solution, known as sliding motion, can be heuristically seen as associated with an infinite number of switchings between the two different system configurations.

For the system evolution to be constrained onto the switching hyperplane, $S$, the gradient of the system vector field must point towards $S$ itself on both sides of it. Hence, by studying the gradient of the vector field in a neighborhood of the switching hyperplane, it is possible to isolate regions on $S$ where sliding motion can occur. In particular, the state trajectories will point towards $S$ if $y>0$ and $\dot{y}<0$ or if $y<0$ and $\dot{y}>0$. By using the system Eqs. (1)-(3), the equivalent reaching condition $y \dot{y}<0$ can be rewritten as

$$
C x C(A x+B u)<0, \quad \forall x \in \mathcal{R} \backslash S .
$$

A system trajectory that hits $S$ at a point where this condition is satisfied on both of its sides is then constrained to evolve along $S$ itself. This gives rise to a "sliding motion" which ends whenever the direction of the vector field on one side of the switching hyperplane or the other changes.

A common approach for the analysis of a system under sliding mode is the equivalent control method by Utkin [1992]. The equivalent control $u_{\text {eq }} \in(-1,1)$ is defined as the control action that keeps the state trajectory on the switching hyperplane. It is hence the control input that gives $\dot{y}=0$ under the constraint $y=0$. This means that, given the assumption that $C B=b_{1} \neq 0$,

$$
u_{\mathrm{eq}}=-(C B)^{-1} C A x \text {. }
$$


By substituting (11) into (1), we obtain the system

$$
\dot{x}=\hat{A} x
$$

where $\hat{A}=\left[I-(C B)^{-1} B C\right] A$ and $I$ denotes the $n \times n$ identity matrix. Using (4) and noting that under sliding mode $\dot{y}=C \dot{x}=\dot{x}^{(1)}=0$, we get the dynamical system

$$
\left(\begin{array}{c}
\dot{x}^{(2)} \\
\dot{x}^{(3)} \\
\vdots \\
\dot{x}^{(n-1)} \\
\dot{x}^{(n)}
\end{array}\right)=\left(\begin{array}{ccccc}
-b_{2} / b_{1} & 1 & 0 & \ldots & 0 \\
-b_{3} / b_{1} & 0 & 1 & & 0 \\
\vdots & & & \ddots & \vdots \\
-b_{n-1} / b_{1} & 0 & 0 & & 1 \\
-b_{n} / b_{1} & 0 & 0 & \ldots & 0
\end{array}\right)\left(\begin{array}{c}
x^{(2)} \\
x^{(3)} \\
\vdots \\
x^{(n-1)} \\
x^{(n)}
\end{array}\right)
$$

This system describes the state evolution under sliding motion. Note that its dynamics are determined by the parameters of matrix $B$ in (1), i.e. by the zero dynamics of the linear part of the relay feedback system. The sliding dynamics is therefore unstable if and only if the open-loop zeros of the system are in the right half-plane. Note also that the sliding trajectories are constrained to lie on a codimension-1 hyperplane since $C x=x^{(1)}=0$. Therefore, the dynamical system (13) is of order $(n-1)$. Higher-order sliding modes can be derived similarly, see [Fridman \& Levant, 1996].

The condition for existence of sliding, previously expressed in terms of the vector field, can be reinterpreted in terms of the equivalent control introduced above. It follows that a sliding motion continues only as long as $\left|u_{\text {eq }}\right|<1$, which is equivalent to

$$
\left|(C B)^{-1} C A x\right|<1
$$

with $x \in S$. In what follows we will refer to the region $\hat{S} \subset S$ where (14) is satisfied as the sliding region:

$$
\hat{S}=\{x \in S:|C A x|<C B\} .
$$

The sliding region is thus nonempty if $C B>0$.

\subsection{Third-order example}

As an illustrative example, we will consider throughout this paper a third-order relay feedback system described by the matrices:

$$
\begin{aligned}
& A=\left(\begin{array}{ccc}
-(2 \zeta \omega+\lambda) & 1 & 0 \\
-\left(2 \zeta \omega \lambda+\omega^{2}\right) & 0 & 1 \\
-\lambda \omega^{2} & 0 & 0
\end{array}\right), \\
& B=\left(\begin{array}{c}
k \\
2 k \sigma \rho \\
k \rho^{2}
\end{array}\right), \quad C=\left(\begin{array}{l}
1 \\
0 \\
0
\end{array}\right)^{T},
\end{aligned}
$$

which corresponds to the transfer function

$$
G(s)=k \frac{s^{2}+2 \sigma \rho s+\rho^{2}}{\left(s^{2}+2 \zeta \omega s+\omega^{2}\right)(s+\lambda)} .
$$

Here $\omega$ and $\zeta$ denote the natural frequency and the damping of the complex pair of poles while $\rho$ and $\sigma$ represent the corresponding quantities for the complex pair of zeros; $-\lambda$ is the location of the real pole; and $k \rho^{2} /\left(\omega^{2} \lambda\right)$ is the steady-state gain. We assume that $k$ and $\lambda$ are positive. Despite its simple structure, this system will be shown to exhibit a particularly rich dynamical behavior.

From (16), it follows that the necessary condition for sliding given in (14) is simply

$$
\left|x^{(2)}\right|<k \text {. }
$$

The sliding region (15) is thus given by $\hat{S}=\{x \in$ $\left.\mathcal{R}^{3}: x^{(1)}=0,\left|x^{(2)}\right|<k\right\}$, which is a strip on the switching hyperplane $S$.

Moreover, the reaching condition (10) becomes

$$
x^{(1)}\left[-(2 \zeta \omega+\lambda) x^{(1)}+x^{(2)}+k u\right]<0 .
$$

Note that Eq. (18) does not depend on $x^{(3)}$, hence the projections of the reaching regions on the plane $\left(x^{(1)}, x^{(2)}\right)$ are given by:

$R_{1}=\left\{\left(x^{(1)}, x^{(2)}\right): x^{(1)}>0, x^{(2)}<\left[(2 \zeta \omega+\lambda) x^{(1)}+k\right]\right\}$,

and

$R_{2}=\left\{\left(x^{(1)}, x^{(2)}\right): x^{(1)}<0, x^{(2)}>\left[(2 \zeta \omega+\lambda) x_{1}-k\right]\right\}$.

Unless stated otherwise, the parameter values are set to be $\rho=\omega=\zeta=\lambda=\kappa=-\sigma=1$. As illustrated in Fig. 1, for these parameter values, we have $R_{1}=\left\{x^{(1)}>0, x^{(2)}<x^{(1)}+1\right\}$ and $R_{2}=\left\{x^{(1)}<0, x^{(2)}>x^{(1)}-1\right\}$. Figure 1 also shows that the sliding region corresponds in this case to the strip $\left|x^{(2)}\right|<k=1$. Within this 


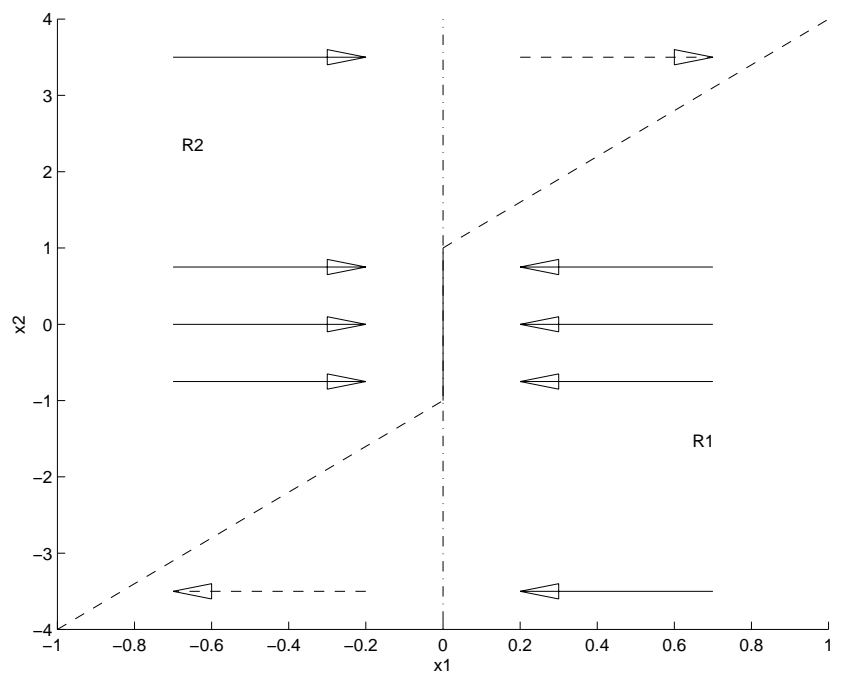

Fig. 1. Projection of the reaching regions and of the sliding strip in the $\left(x^{(1)}, x^{(2)}\right)$ plane for the third-order example. Arrows indicate whether or not the trajectory point towards the switching hyperplane. The switching hyperplane $S$ is marked by a dashed-dotted line and the sliding strip $\hat{S}$ with continuous line. The reaching regions $R_{1}$ and $R_{2}$ define where a solution may enter the sliding strip and they are defined by $S$ and the dashed lines. Note that the solutions leave the strip as $\left|x^{(2)}\right|=k=1$.

region the system evolution is described by a reduced-order model of the form, according to (13):

$$
\left(\begin{array}{c}
\dot{x}^{(2)} \\
\dot{x}^{(3)}
\end{array}\right)=\left(\begin{array}{cc}
-2 \sigma \rho & 1 \\
-\rho^{2} & 0
\end{array}\right)\left(\begin{array}{c}
x^{(2)} \\
x^{(3)}
\end{array}\right) \text {. }
$$

Note that the dynamics of (19) depends on the position of the zeros of the linear part of the relay feedback system (i.e. depend on the parameters of $B$ in (16)). Hence, the sliding dynamics are unstable if the product $\sigma \rho$ is negative. Unstable zero dynamics will be shown to play an important role in the formation of periodic solutions with sliding.

\section{Periodic Solutions}

Relay feedback systems typically exhibit selfoscillations [Tsypkin, 1984]. Namely, the system evolution is attracted to a stable limit cycle which is commonly characterized as follows (see Fig. 2 for a numerical example). Starting from a point $x_{0} \in S \backslash \hat{S}$ the state trajectory crosses the switching plane at a point $x_{1} \in S \backslash \hat{S}$. Here the system changes its configuration and then evolves on the other side of the switching hyperplane until reaching again the point $x_{0}$.

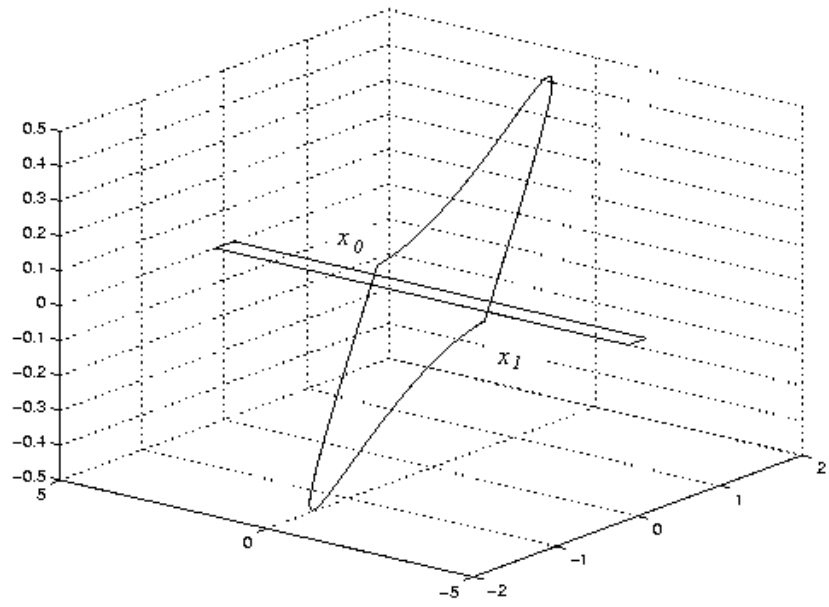

Fig. 2. Simple orbit for the third-order example with $\rho=3$. The orbit does not intersect with the sliding strip $\hat{S}$, which is indicated as a rectangle.

Consider a solution of the relay feedback system (1)-(3) that starts at $x_{0}$ with $C x_{0}=0$ and $C A x_{0}>C B$. Solving the system equations, the next switching plane intersection is then given by:

$$
x_{1}=e^{A \delta_{01}} x_{0}-\left(e^{A \delta_{01}}-I\right) A^{-1} B,
$$

where the switching time $\delta_{01}$ depends on $x_{0}$ and is defined as the smallest positive value such that the right-hand side of $(20)$ is orthogonal to $S$, i.e. $C x_{1}=0$. Assume that $C A x_{1}<-C B$. The next intersection is then given by

$$
x_{2}=e^{A \delta_{12}} x_{1}+\left(e^{A \delta_{12}}-I\right) A^{-1} B,
$$

with $C x_{2}=0$. Similarly, under the assumption that $\left|C A x_{k}\right|>C B$, we define the intersection $x_{k+1}$ and switching time $\delta_{k, k+1}$ for all $k \geq 0$.

This type of trajectory is a periodic solution of the relay feedback system under investigation if there exists a positive integer $P$ such that $x_{2 P}=x_{0}$. An orbit is called one-periodic if $x_{2}=x_{0}$, twoperiodic if $x_{4}=x_{0}$, but $x_{2} \neq x_{0}$ etc. A periodic solution of period $T$ is symmetric if $x^{*}(t)=$ $-x^{*}(t+T / 2)$ for all $t>0$. In what follows, oneperiodic solutions evolving outside of the sliding region will also be called simple orbits.

\subsection{Poincaré maps}

In order to characterize the existence and stability of simple orbits, we now introduce some appropriate discrete-time maps.

The upper switching map is defined as the map $\Pi^{+}: S \backslash \hat{S} \mapsto S$ that describes the system dynamics 
from $x_{0}$ to $x_{1}$ as discussed previously. This map is thus given by

$$
\Pi^{+}\left(x_{0}\right)=N\left(\delta_{01}\right) x_{0}-M\left(\delta_{01}\right),
$$

where

$$
\begin{aligned}
& N(\delta)=\exp (A \delta), \\
& M(\delta)=[N(\delta)-I] A^{-1} B,
\end{aligned}
$$

and $\delta_{01}=\delta_{01}\left(x_{0}\right)$ is the smallest solution of the switching condition:

$$
C\left[N\left(\delta_{01}\right) x_{0}-M\left(\delta_{01}\right)\right]=0 .
$$

Note that the value set of $\Pi^{+}$is the whole switching hyperplane $S$.

Similarly, the lower switching map $\Pi^{-}: S \backslash \hat{S} \mapsto$ $S$ is defined as the map

$$
\Pi^{-}\left(x_{1}\right)=N\left(\delta_{12}\right) x_{1}-M\left(\delta_{12}\right),
$$

that describes the system evolution from $x_{1}$ to $x_{2}$. Finally, we define the switching map as the composition of $\Pi^{+}$and $\Pi^{-}$:

$$
\Pi=\Pi^{-} \circ \Pi^{+} .
$$

This Poincaré map is an $(n-1)$-dimensional mapping of the switching hyperplane back to itself. Analytically, (22) and (24) are given in terms of the $n$-dimensional state vector $x$ with the additional constraints defining the switching intersections. Since the first component of the state on $S$ is identically zero, these equations will implicitly define an $(n-1)$-dimensional map as expected.

\subsection{Existence and local stability}

Using the map defined above, a necessary condition for the existence of a simple symmetric orbit is that

$$
\Pi^{+}\left(x_{0}\right)=-x_{0},
$$

which is equivalent to

$$
N\left(\delta_{01}\right) x_{0}-M\left(\delta_{01}\right)=-x_{0} .
$$

Hence, the necessary conditions of existence of a symmetric simple orbit is that there exists $\delta>0$ solving the scalar equation

$$
C[I+N(\delta)]^{-1} M(\delta)=0 .
$$

This condition is not sufficient, because Eq. (27) can have solutions that do not correspond to actual orbits of the system. Note that because of the symmetry a condition equivalent to (27) is obtained if the same argument is repeated using the lower switching map $\Pi^{-}$instead of $\Pi^{+}$.

Consider a solution $\bar{\delta}$ of (27) that corresponds to an actual symmetric simple orbit of the system and let $\bar{x}_{0}$ be the corresponding fixed point of the upper switching map $\Pi^{+}$. The local stability properties of the orbit are then given by the eigenvalues of the Jacobian of $\Pi^{+}$for $\delta=\bar{\delta}$ and $x_{0}=\bar{x}_{0}$. Using implicit differentiation this Jacobian can be derived as

$$
D \Pi^{+}=\bar{N}\left(I-\frac{\dot{x}_{0}^{+} C \bar{N}}{C \dot{x}_{1}^{-}}\right),
$$

where $\bar{N}=N(\bar{\delta}), \dot{x}_{0}^{+}=A \bar{x}_{0}-B$ and $\dot{x}_{1}^{-}=\bar{N} \dot{x}_{0}^{+}$. Compare similar derivations in [di Bernardo \& Vasca, 1999; Åström, 1995; Johansson et al., 1999].

Asymmetric one-periodic orbits may also be studied in a similar way by replacing condition (25) with the more general one:

$$
\Pi\left(x_{0}\right)=\left(\Pi^{-} \circ \Pi^{+}\right)\left(x_{0}\right)=x_{0} .
$$

The necessary condition of existence, corresponding to (27), follows in this case from (29) together with the constraints $C x_{0}=0$ and $C x_{1}=0$. Specifically, we get the condition that there should exist $\delta_{01}, \delta_{10}>0$ such that

$$
\begin{aligned}
& C\left[I-N\left(\delta_{01}+\delta_{10}\right)\right]^{-1}\left[N\left(\delta_{01}+\delta_{10}\right)\right. \\
& \left.\quad-2 N\left(\delta_{01}\right)+I\right] A^{-1} B=0, \\
& C\left[I-N\left(\delta_{01}+\delta_{10}\right)\right]^{-1}\left[N\left(\delta_{01}+\delta_{10}\right)\right. \\
& \left.\quad-2 N\left(\delta_{10}\right)+I\right] A^{-1} B=0 .
\end{aligned}
$$

The stability of a generic orbit is then given by the Jacobian

$$
D \Pi=D \Pi^{-} \cdot D \Pi^{+} .
$$

From the symmetry of the relay feedback system discussed in Sec. 2, it follows that asymmetric orbits come always in pairs. This can be shown using the following argument. If $x^{*}:[0, \infty) \mapsto \mathcal{R}^{n}$ is a periodic solution of the system, then $x^{*}$ satisfies equations (1)-(3), i.e. it satisfies $\dot{x}=A x-$ $B \operatorname{sgn}(C x)$. If this is true, then

$$
\begin{aligned}
-\dot{x}^{*} & =A\left(-x^{*}\right)-B \operatorname{sgn}\left(C\left(-x^{*}\right)\right) \\
& =-\left[A x^{*}-B \operatorname{sgn}\left(C x^{*}\right)\right],
\end{aligned}
$$


thus also $-x^{*}$ satisfies the system equations and hence is a periodic solution of (1) and (2). Hence, for each asymmetric orbit, $x^{*}$, there is another asymmetric solution, $\hat{x}=-x^{*}$, which can be obtained by reflecting each point of $x^{*}$ around the origin. If the first orbit intersects the switching hyperplane in $x_{0}$ and $x_{1}$, say, the second orbit will then have intersections $-x_{0}$ and $-x_{1}$. From a similar argument, it also follows that every one-periodic orbit is symmetric if $x_{1}=-x_{0}$.

The existence and stability conditions reported above can be easily generalized to $P$-periodic orbits with $P>1$.

\section{Symmetry-Breaking Bifurcation}

It has been often assumed in the control literature that self-oscillations of symmetric, unforced relay feedback systems such as (1) and (2) are also symmetric. Specifically, it is usually conjectured that asymmetric periodic solutions in these systems can only exist either by means of some type of external forcing term acting on the system or because of an intrinsic asymmetric relay characteristic (see e.g. [Tsypkin, 1984], p. 179).

Given that the relay feedback systems under investigation are unforced and symmetric, it is quite tempting to conclude that asymmetric orbits cannot exist. Unexpectedly, this is only true for a restricted class of relay systems. For instance, in the appendix it is shown that relay feedback systems with linear dynamics having real stable poles and no zeros do not have asymmetric orbits.

In what follows we show via a counterexample that, contrary to what is usually assumed, symmetric and unforced relay feedback systems can indeed exhibit asymmetric periodic solutions. This is done by locating a symmetry-breaking bifurcation point of a symmetric simple orbit. As reported in the literature [Kuznetsov, 1995], the transition from a symmetric solution to a pair of conjugate asymmetric orbits is, in fact, observed at this bifurcation point.

As pointed out by Kuznetsov [1995], the possible bifurcations of the symmetric periodic solutions exhibited by a symmetric system cannot be studied using the global Poincaré map from the switching plane back to itself along the entire orbit. For symmetric systems, this map is, in fact, the composition of two identical submappings or equivalently the second iterate of the same map. This has been proven for smooth dynamical systems in [Swift \& Wiesenfeld, 1984] and is here extended to the case of relay feedback systems.

Consider a simple periodic limit cycle of pe$\operatorname{riod} 2 \delta$ and the previously defined global map $\Pi=$ $\Pi^{-} \circ \Pi^{+}$, where $\Pi^{-}$and $\Pi^{+}$are the mappings describing the system evolution above and below the switching hyperplane, respectively. Because of the symmetry of the system, it is easy to verify that

$$
\Pi^{-}=(-I) \circ \Pi^{+} \circ(-I) .
$$

Hence, we get

$$
\begin{aligned}
\Pi & =(-I) \circ \Pi^{+} \circ(-I) \circ \Pi^{+} \\
& =\left(-I \circ \Pi^{+}\right)^{2}:=(\tilde{\Pi})^{2} .
\end{aligned}
$$

Note that any one-periodic solution corresponds to a fixed point of $\Pi$, while only symmetric orbits correspond to fixed points of the half-map $\tilde{\Pi}$. Thus, a symmetric solution undergoes a symmetrybreaking bifurcation when $\tilde{\Pi}$ undergoes a flip bifurcation, i.e. when the Jacobian of $\tilde{\Pi}$ has a multiplier at -1 . When this occurs, the symmetric orbit associated with a fixed point of $\tilde{\Pi}$ bifurcates into an asymmetric solution associated with a twoperiodic cycle of $\tilde{\Pi}$. This in turn corresponds to a one-periodic asymmetric orbit of the relay feedback system and hence a fixed point of the global map $\Pi$.

To locate the symmetry-breaking bifurcation point we need the Jacobian DĨ. This can be obtained using the method presented above. For third-order examples, the bifurcation point can be derived from the necessary condition of existence for a simple symmetric limit cycles (27) together with the condition for a flip bifurcation [Kuznetsov, 1995]

$$
\operatorname{det}(L D \tilde{\Pi})+\operatorname{tr}(L D \tilde{\Pi})+1=0
$$

where $L$ is a projection matrix, which extracts the two-dimensional nontrivial matrix from the Jacobian $D \tilde{\Pi}$. This analytical set of conditions can then be solved w.r.t. the system parameters to obtain the exact value at which the symmetry-breaking bifurcation occurs.

We will now detail this procedure to the case of the third-order relay feedback system described in Sec. 2.2. 


\subsection{Asymmetric orbits of an unforced, symmetric relay feedback system}

Consider the third-order system described in Sec. 2 . We restrict our attention to the codimension-one bifurcations observed when the parameter $\rho$ is varied, while the other parameters are kept constant at $\omega=-\zeta=-\lambda=k=\sigma=1$.

The analytical necessary condition for the existence of a simple orbit (27) and the flip condition for $\tilde{\Pi}(36)$ were solved with respect to the parameter $\rho$, using the algebraic manipulation package Maple. The flip bifurcation of the half-map was located at $\rho \approx 0.694$. At this point the Jacobian of $\tilde{\Pi}$ has a multiplier crossing the unit circle at -1 . Therefore, the orbit undergoes a symmetry-breaking bifurcation. This is in perfect agreement with the numerical simulations, which are presented next.

An example of an asymmetric orbit is depicted in Fig. 3, where the orbit for $\rho=0.6$ is given. The corresponding plot in the time domain is shown in Fig. 4. As outlined above, each asymmetric orbit is characterized by a corresponding two-periodic cycle $\left(x_{0}, x_{1}\right)$ of $\tilde{\Pi}$. For $\rho=0.6$, we have

$$
x_{0} \approx\left(\begin{array}{c}
0 \\
1.779 \\
-1.194
\end{array}\right), \quad x_{1} \approx\left(\begin{array}{c}
0 \\
-1.027 \\
-1.343
\end{array}\right)
$$

The periodic solution spends $\delta_{01} \approx 0.915$ in the lower section of the phase space and $\delta_{10}=\delta_{12} \approx$ 2.808 in the upper part, with $T=\delta_{01}+\delta_{10}=3.723$ being the period of the orbit.

Figure 5 shows that, as predicted by the theory, the bifurcation point is indeed at $\rho \approx 0.69$. In this figure the values of $\delta_{01}$ and $\delta_{10}$ are presented for different values of $\rho$. We see that the system spends the same amount of time above and below the switching hyperplane when $\rho>0.694$. As $\rho$ is decreased past the symmetry-breaking bifurcation, symmetric solutions branch out into asymmetric orbits. Note that the period of the orbit $T=\delta_{01}+\delta_{10}$ increases as $\rho$ is decreased. Also, a sudden change in the rate of change of $T$ can be observed, as $\rho$ passes through the bifurcation point, see Fig. 6. Details of the symmetry-breaking bifurcation are given in Table 1 , where the switching times $\delta_{01}$ and $\delta_{10}$ are given together with the first two components of the

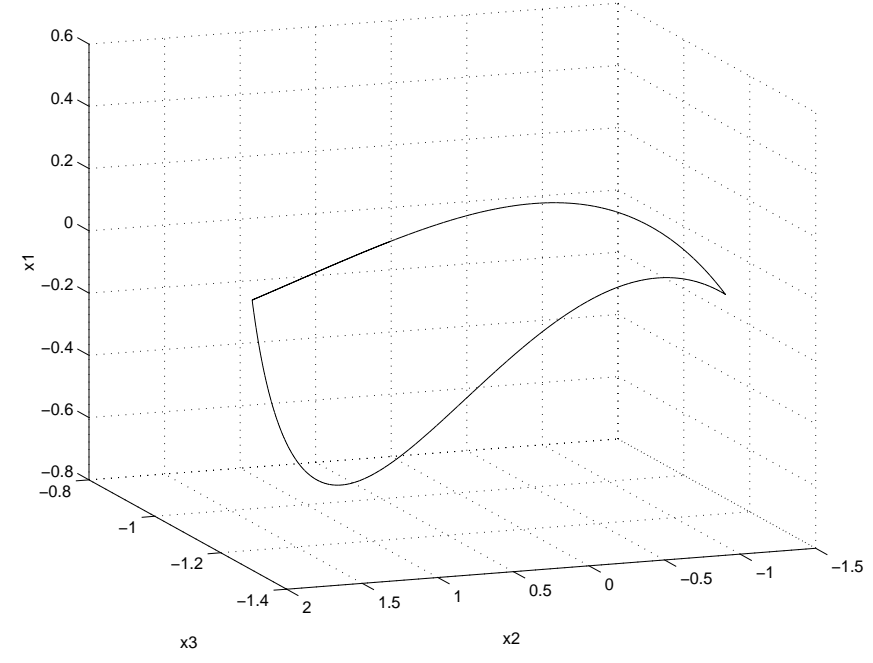

Fig. 3. Asymmetric simple orbit for $\rho=0.6$. This orbit appears as $\rho$ is further decreased after the symmetry-breaking bifurcation has taken place $\rho \approx 0.694$. The orbit is unstable.
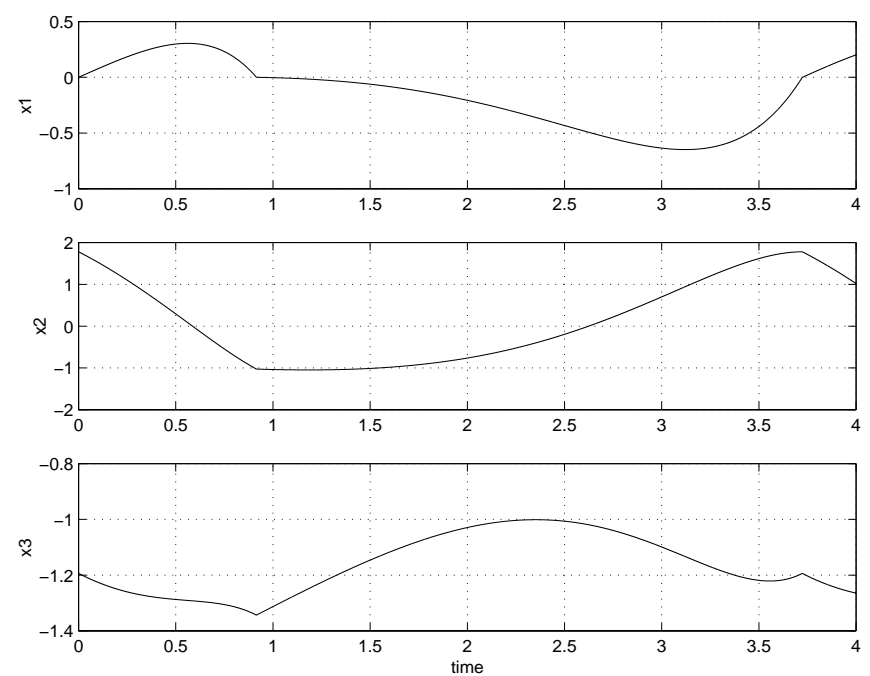

Fig. 4. Time evolution of the asymmetric periodic solution shown in previous figure.

hyperplane intersections $\left(x_{0}^{(2)}, x_{0}^{(3)}\right)$ and $\left(x_{1}^{(2)}, x_{1}^{(3)}\right)$, as functions of the bifurcation parameter $\rho$.

Because of the symmetry of the system, following the argument presented in Sec. 3, it is possible to show that for each asymmetric solution there also exists a corresponding conjugate solution. The structure of these solutions is, of course, identical. The only difference is that their intersection points with the switching plane are reflected around the origin.

Note that the asymmetric solutions we analyzed originate at a symmetry-breaking bifurcation of an unstable periodic solution. Thus, they are also 


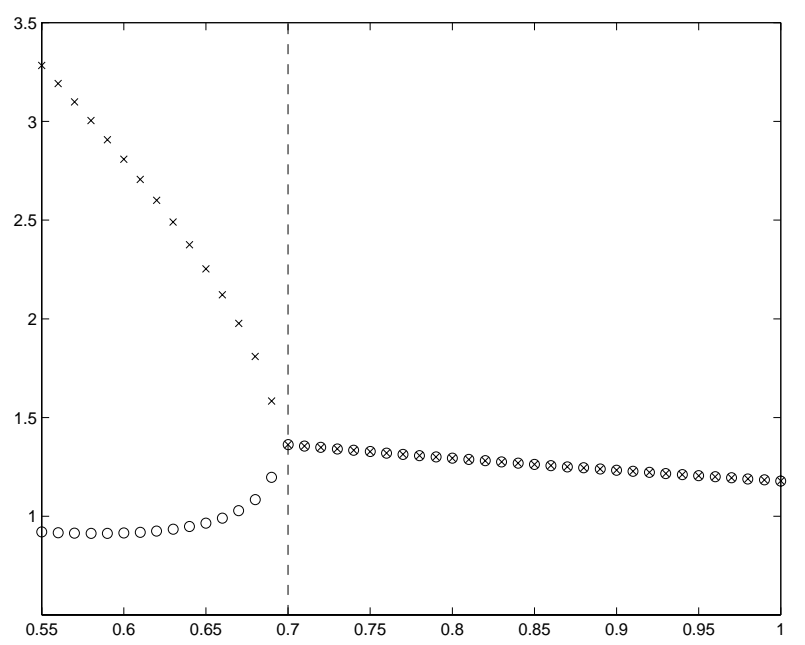

Fig. 5. Bifurcation diagram illustrating how the symmetry breaks at $\rho \approx 0.694$. The switching time $\delta_{01}$ (circles) spent by the system below the switching hyperplane and time $\delta_{10}$ (crosses) spent above are shown as functions of $\rho$. For decreasing values of $\rho$, we notice that after the symmetrybreaking bifurcation at $\rho \approx 0.694$ a clear separation of the two components of the period time $T=\delta_{01}+\delta_{10}$ can be observed. The diagram agrees with the theoretical argument reported in the paper.

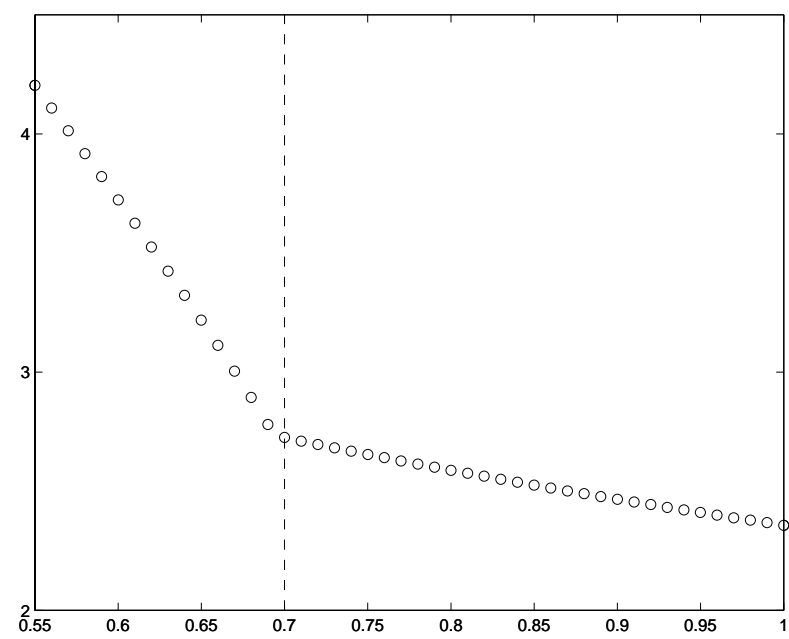

Fig. 6. Period time $T=\delta_{01}+\delta_{10}$ corresponding to the bifurcation diagram in previous figure. Note that to the left of the symmetry-breaking bifurcation a steeper increase of the period can be observed for decreasing values of $\rho$ than to the right of the point.

unstable. Instances of stable asymmetric solutions will be reported later in the paper (see for instance Fig. 16).

\section{Sliding Orbits}

Recently, relay feedback systems have been shown to exhibit periodic solutions lying partly within the

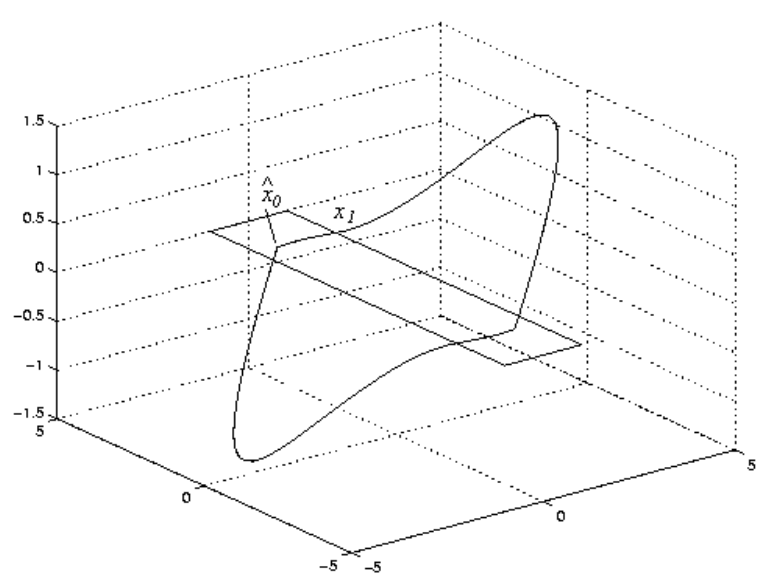

Fig. 7. Sliding orbit for $\rho=1$. Note how the solution enters the sliding strip when $x^{(1)}=0$ and $\left|x^{(2)}\right|<k=1$ and leaves the strip at its boundary.

sliding region $\hat{S}=\{x \in S:|C A x|<C B\}$. An example of such a sliding orbit for the example described in Sec. 2.2 is shown in Fig. 7.

A general relay feedback system with sliding orbits can be analyzed by the introduction of an additional Poincaré map which we term as sliding map. In what follows, we first define this map and then detail its application to characterize the existence and stability of sliding orbits. As shown in Sec. 6, this map is also an invaluable tool to understand the bifurcation scenario through which these solutions are formed.

\subsection{Sliding map}

Consider a relay feedback system (1)-(3) with nonempty sliding strip $\hat{S}$ and with unstable zero dynamics and define the boundary $\partial \hat{S}$ of $\hat{S}$ as:

$$
\partial \hat{S}=\{x \in S:|C A x|=C B\} .
$$

Then the solution through a point $\hat{x}_{0} \in \hat{S} \backslash\{0\}$ will eventually end up on the boundary of $\hat{S}$ in a point $x_{1}$, say. The sliding map $\Sigma: \hat{S} \mapsto \partial \hat{S}$ can be defined as the map from $\hat{x}_{0} \in \hat{S}$ to $x_{1} \in \partial \hat{S}$. This can be derived analytically using the reduced-order model (13) describing the system dynamics within the sliding region. The map is given by

$$
\Sigma: \hat{x}_{0} \mapsto x_{1}=\exp \left(\hat{A} \delta_{s}\right) \hat{x}_{0} .
$$

Note that $x^{(1)} \equiv 0$ during sliding and the time $\delta_{s}>0$ is the time for the trajectory to reach the boundary of the sliding strip, i.e. from (14), the smallest solution to the equation

$$
C A \exp (\hat{A} \delta) \hat{x}_{0} \pm C B=0 .
$$


Table 1. The characteristics of an unstable simple orbit undergoing a symmetry-breaking bifurcation at $\rho \approx$ 0.69399. The table presents the bifurcation parameter $\rho$; the switching times $\delta_{01}$ and $\delta_{10}$ spent by the system below and above the switching hyperplane, respectively; and the coordinates of the switching plane intersections $\left(x_{0}^{(2)}, x_{0}^{(3)}\right)$ and $\left(x_{1}^{(2)}, x_{1}^{(3)}\right)$. For $\rho>0.69399$, it holds that $\delta_{01}=\delta_{10}$ and $x_{1}=-x_{0}$, so the orbit is symmetric. After the symmetry-breaking bifurcation, for $\rho<0.69399$, the switching times become distinct and $x_{1} \neq-x_{0}$. For $\rho<0.58$, the asymmetric orbit intersects with the switching plane, forming asymmetric sliding orbits. This follows from that $x_{0}^{(2)}$ becomes greater than -1 .

\begin{tabular}{|c|c|c|c|c|}
\hline$\rho$ & $\delta_{01}$ & $\delta_{10}$ & $\left(x_{0}^{(2)}, x_{0}^{(3)}\right)$ & $\left(x_{1}^{(2)}, x_{1}^{(3)}\right)$ \\
\hline 0.74 & 1.334136107 & 1.334136107 & {$[-1.596828217,-0.1684077405]$} & {$[1.596828217,0.168407742]$} \\
\hline 0.73 & 1.341117136 & 1.341117136 & {$[-1.592698541,-0.1591870604]$} & {$[1.592698545,0.159187054]$} \\
\hline 0.72 & 1.348187453 & 1.348187453 & {$[-1.588545257,-0.1500066710]$} & {$[1.588545255,0.150006673]$} \\
\hline 0.71 & 1.355348998 & 1.355348998 & {$[-1.584368094,-0.1408669791]$} & {$[1.584368092,0.140866979]$} \\
\hline 0.70 & 1.362603774 & 1.362603774 & {$[-1.580166783,-0.1317683720]$} & {$[1.580166780,0.131768378]$} \\
\hline 0.69399 & 1.361100308 & 1.372957117 & {$[-1.574129658,-0.139084707]$} & {$[1.581109276,-0.113557108]$} \\
\hline 0.69 & 1.196769423 & 1.583473016 & {$[-1.453488234,-0.5303070661]$} & {$[1.67662885,-0.282994287]$} \\
\hline 0.68 & 1.084698576 & 1.809028995 & {$[-1.337481777,-0.8368945855]$} & {$[1.735794026,-0.602322690]$} \\
\hline 0.67 & 1.027580879 & 1.976736076 & {$[-1.262674373,-1.004213892]$} & {$[1.761227586,-0.781650870]$} \\
\hline 0.66 & 0.9908473568 & 2.121537901 & {$[-1.206147750,-1.114831359]$} & {$[1.774553344,-0.9036940843]$} \\
\hline 0.65 & 0.9653958793 & 2.252856916 & {$[-1.161093080,-1.192307961]$} & {$[1.781562918,-0.9921305228]$} \\
\hline 0.64 & 0.9472370999 & 2.374959760 & {$[-1.124181633,-1.247637785]$} & {$[1.784773662,-1.058047930]$} \\
\hline 0.63 & 0.9342215855 & 2.490238664 & {$[-1.093434391,-1.287055716]$} & {$[1.785508850,-1.107757003]$} \\
\hline 0.62 & 0.9250466930 & 2.600211120 & {$[-1.067542063,-1.314503950]$} & {$[1.784541557,-1.145262008]$} \\
\hline 0.61 & 0.9188598450 & 2.705921692 & {$[-1.045578327,-1.332655773]$} & {$[1.782355099,-1.173287554]$} \\
\hline 0.60 & 0.9150717832 & 2.808132799 & {$[-1.026856953,-1.343415168]$} & {$[1.779266241,-1.193780454]$} \\
\hline 0.59 & 0.9132583337 & 2.907426174 & {$[-1.010852259,-1.348188629]$} & {$[1.775489965,-1.208183220]$} \\
\hline 0.58 & 0.9131043811 & 3.004261512 & {$[-0.9971511027,-1.348045022]$} & {$[1.771176121,-1.217595222]$} \\
\hline 0.57 & 0.9143698824 & 3.099012607 & {$[-0.9854221520,-1.343815332]$} & {$[1.766431382,-1.222873441]$} \\
\hline 0.56 & 0.9168682213 & 3.191990793 & {$[-0.9753952677,-1.336157832]$} & {$[1.761332985,-1.224698431]$} \\
\hline 0.55 & 0.9204518658 & 3.283460791 & {$[-0.9668471353,-1.325602282]$} & {$[1.755937649,-1.223619127]$} \\
\hline
\end{tabular}

Simple orbits with no sliding are described by $\Pi=\Pi^{-} \circ \Pi^{+}$, as discussed in Sec. 3. Sliding orbits, as the one depicted in Fig. 7, are instead described by a suitable composition of $\Pi^{+}, \Pi^{-}$and $\Sigma$. The composition is given by the map $\Xi$ defined as

$$
\partial \hat{S} \mapsto \partial \hat{S}
$$

defined as:

$$
\Xi=\Xi^{-} \circ \Xi^{+}=\left(\Sigma \circ \Pi^{-}\right) \circ\left(\Sigma \circ \Pi^{+}\right),
$$

with the constraint that the value sets of $\Pi^{+}$and $\Pi^{-}$are restricted to $\hat{S}$. For example, starting from a point $x_{0} \in S \backslash \hat{S}$ we have $\Pi^{+}: x_{0} \mapsto \hat{x}_{0}$, with $\hat{x}_{0} \in \hat{S} \backslash\{0\}$ and $\Sigma: \hat{x}_{0} \mapsto x_{1}$, with $x_{1} \in \partial \hat{S}$. Thus, we have

$$
\Xi^{+}: x_{0} \mapsto x_{1}=\exp \left(\hat{A} \delta_{s}\right)\left[N\left(\delta_{01}\right) x_{0}-M\left(\delta_{01}\right)\right],
$$

where the variables $\delta_{01}$ and $\delta_{s}$ are given as in (23) and (39), respectively.

\subsection{Existence and local stability}

The map $\Xi$ and its submappings $\Xi^{-}$and $\Xi^{+}$can be used to derive necessary conditions for existence of sliding orbits, similarly to what done with the switching map $\Pi$ for orbits without sliding. For example, fixed points of $-I \circ \Xi^{+}$define symmetric one-periodic orbits with sliding. These are obtained by solving the equation $\Xi^{+}\left(x_{0}\right)=-x_{0}$ with the constraint that $C \hat{x}_{0}=0$. This yields that $\delta_{01}, \delta_{s}>0$, as given by (23)-(39), should fulfill the necessary conditions:

$$
C\left[\exp \left(\hat{A} \delta_{s}\right) N\left(\delta_{01}\right)+I\right]^{-1} \exp \left(\hat{A} \delta_{s}\right) M\left(\delta_{01}\right)=0,
$$




$$
\begin{aligned}
& C A\left[\exp \left(\hat{A} \delta_{s}\right) N\left(\delta_{01}\right)+I\right]^{-1} \exp \left(\hat{A} \delta_{s}\right) M\left(\delta_{01}\right) \\
& \quad \pm C B=0
\end{aligned}
$$

Stability of sliding orbits can be studied by computing the Jacobian of the mapping $\Xi$ similarly to what done for $\Pi$ in the nonsliding case (see Sec. 3). The only difference is that now $\Xi$ is the composition of $\Pi^{+}, \Pi^{-}$and the sliding map $\Sigma$ and thus one must consider the contribution of the sliding map in the Jacobian derivation. $P$-periodic orbits with sliding are identified by fixed points of $\left(\Xi^{+} \circ \Xi^{-}\right)^{P}$.

\subsection{Numerical evidence}

Consider again the third-order example with $\omega=$ $\zeta=\lambda=k=-\sigma=1$. Sliding orbits exist for several values of the bifurcation parameter $\rho$. The stable sliding orbit observed when $\rho=1$ is depicted in Fig. 7. Here we see that the state evolution hits the switching plane within the sliding strip, where the necessary condition for sliding $\left|x^{(2)}\right|<1$ is satisfied. The state then evolves within the strip according to the reduced-order model (19) until eventually crossing the boundary of $\hat{S}$ at $\left|x^{(2)}\right|=1$. When this occurs, the state evolution leaves the switching hyperplane. Thus, as shown in Fig. 7, a periodic connection links the sliding region back with itself, giving rise to a sliding orbit. Note that this is possible because of the unstable sliding dynamics. If the parameters were chosen so that the dynamics (19) were stable, the sliding trajectory would instead evolve towards the stable attractor in the origin of the switching hyperplane and no periodic connection would occur. In this case, the sliding map $\Xi$ is a one-dimensional map since the thirdorder model under investigation is characterized by a planar sliding region. This simplifies the analysis of sliding orbits considerably.

In Table 1, we can see that sliding orbits exist also for $\rho \leq 0.58$. Specifically, the table shows that the switching plane intersection of the orbit, $x_{0}$, moves towards the boundary of the sliding strip as $\rho$ decreases. At $\rho \approx 0.58$ the orbit intersection enters the boundary of the sliding strip $\left(\left|x_{0}^{(2)}\right|<1\right)$. This causes the formation of a periodic solution characterized by a sliding section. As will be shown in the next section, this mechanism represents a more general bifurcation event, which we shall term as "sliding bifurcation".

\section{Sliding Bifurcation}

To uncover the mechanism underlying the formation of sliding orbits in relay feedback systems, we now restrict our attention to the third-order example presented in Sec. 2.2. Specifically, we focus onto codimension-one bifurcations of simple periodic orbits of this system observed by fixing the parameters $\omega=\zeta=\lambda=k=-\sigma=1$ and varying $\rho$ in a neighborhood of the value $\rho=1$ at which a sliding orbit was observed (see Fig. 7).

The bifurcation diagram as $\rho$ is varied from -1 to 3 is reported in Fig. 9, where the steady-state

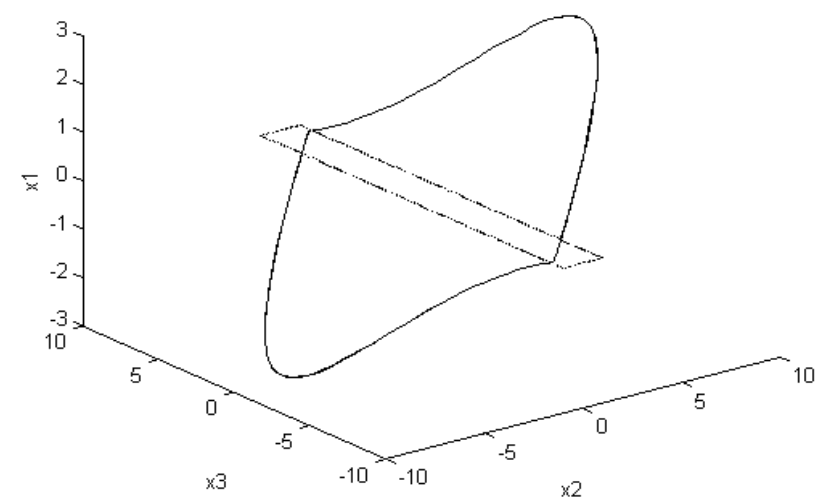

Fig. 8. Simple orbit at the sliding bifurcation point, i.e. $\rho=$ 2.1. Note how the intersections of the orbit with the switching plane lie as expected on the boundary of the sliding strip, $\partial \hat{S}$. Any further parameter variation will cause the formation of a sliding orbit.

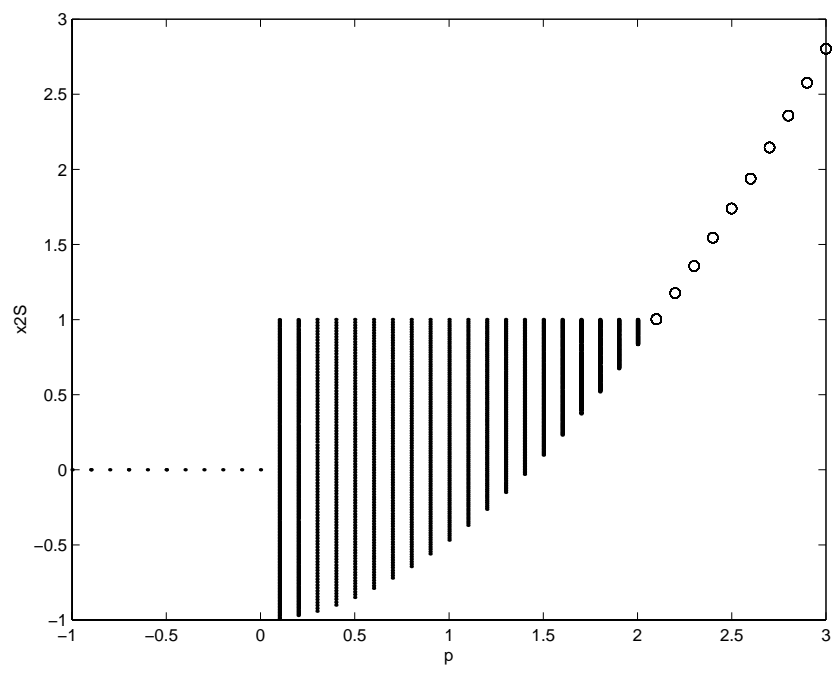

Fig. 9. Diagram showing sliding bifurcation, which takes place at $\rho \approx 2.1$ for decreasing values of $\rho$. The second component of the switching plane intersection $x_{s}^{(2)}$ is shown as a function of $\rho$. The bars indicate sliding segments. For $\rho>2.1$ the system shows a stable periodic solution without sliding and for $-1<\rho<0$ there are no periodic solutions. 
intersections of the second state component with the switching plane $S$, say $x_{s}^{(2)}$, are plotted against $\rho$. The dots for negative values of $\rho$ illustrate that the origin is a global attractor, the bars for $\rho$ in the middle range shows the extension of the sliding segments of the orbits, and the circles for larger values of $\rho$ defines simple orbits (without sliding).

We see that as $\rho$ decreases a nonsliding oneperiodic solution turns into a sliding orbit at $\rho=$ $\rho^{*} \approx 2.1$. As shown in Figs. 8 and 9 , at this specific parameter value the intesection of the orbit with the switching hyperplane crosses the boundary of the sliding region, i.e. $\left|x_{0}^{(2)}\left(\rho^{*}\right)\right|=1$. For lower values of $\rho$, the system evolution is then constrained to "slide" within the strip until reaching its boundary again because of the unstable dynamics of the sliding model $(\sigma \rho<0$ in (19)). This transition, which we term "sliding bifurcation", is locally smooth in the sense that the local mapping around the bifurcation point can be shown to be smooth to arbitrary order and have continuous first derivative [diBernardo et al., in preparation]. The bifurcation only involves one of the eigenvalues of the bifurcating simple orbit to become identically zero (the eigenvalue associated with the eigenvector transversal to the switching plane). Hence, after the bifurcation periodic solutions having the same structure of the bifurcating simple orbits but with an additional sliding section can be observed, see Fig. 9. As $\rho$ is further decreased, a branch of sliding orbits characterized by longer and longer sliding sections is detected until, at $\rho=0$, the origin becomes the only stable attractor.

In the parameter range $\rho \in(0,3)$, solving the necessary conditions of existence of simple orbits presented in Sec. 5 suggests that for each value of $\rho$ there exist two possible periodic solutions, one stable and the other unstable. By decreasing $\rho$, while the stable solution undergoes the sliding bifurcation at $\rho \approx 2.1$ described above, the unstable one persists until at $\rho \approx 0.69$ a family of asymmetric unstable solutions is generated via a symmetry-breaking bifurcation as the one analyzed in Sec. 4. These asymmetric orbits also undergo a sliding bifurcation. This is observed at $\rho \approx 0.58$, as detailed in Table 1. At this bifurcation point, an asymmetric orbit enters the sliding strip, giving rise to an asymmetric sliding orbit.

Further investigations of the system parameter space reveals that the origin remains the only attractor of the system for $\rho \in(-9.4,0)$, as shown in
Fig. 10. Then at $\rho \approx-9.4$, the map $\tilde{\Pi}$ has a multiplier located at +1 . Hence, this bifurcation can be classified as a saddle-node bifurcation and the creation of an unstable and a stable simple orbit should be expected. This has been numerically observed and Fig. 10 shows how the intersection of the stable orbit evolves for $\rho<-9.4$.

The bifurcation mechanism outlined above can also explain the formation of sliding orbits in $n$ dimensional relay feedback systems (and more generally in piecewise smooth dynamical systems with sliding). For these systems, we say that a simple orbit undergoes a sliding bifurcation when its intersection with the switching hyperplane crosses the boundary of the sliding region, as the system parameters are varied. For instance, consider the generic relay feedback system (1)-(3) and let $\rho$ denote the parameter that is varied and $x_{0}=\bar{x}_{0}(\rho) \in$ $S$ be the fixed point of the switching map $\Pi^{+}$corresponding to a generic simple one-periodic orbit of the system. A sliding bifurcation occurs then at $\rho=\rho^{*}$ if $\bar{x}_{0}\left(\rho^{*}\right) \in \partial \hat{S}$. This situation corresponds to the condition

$$
C A \bar{x}_{0}\left(\rho^{*}\right) \pm C B=0,
$$

where $C \bar{x}_{0}\left(\rho^{*}\right)=0$. Note that the system matrices $A, B$ and $C$ can all depend on $\rho$ in the general case. For symmetric one-periodic orbits, it follows from (27) and (45) that a necessary condition for a

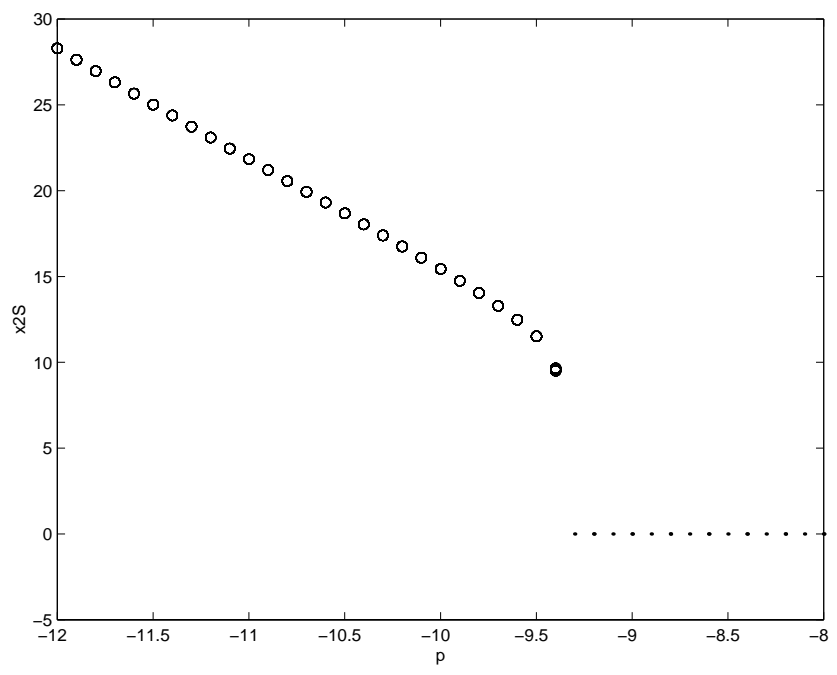

Fig. 10. Bifurcation diagram for $-12<\rho<-8$, compare previous figure. The diagram illustrates the saddle-node bifurcation at $\rho \approx-9.4$, where an unstable and a stable simple are formed for decreasing $\rho$. For $\rho>-9.4$, the origin is a globally stable attractor, while for $\rho<-9.4$ there exists the stable orbit shown here. 
sliding bifurcation to occur is that there should exist a $\delta^{*}>0$ and $\rho^{*}$ such that

$$
\begin{aligned}
C\left[I+N\left(\delta^{*}\right)\right]^{-1} M\left(\delta^{*}\right) & =0 \\
C A\left[I+N\left(\delta^{*}\right)\right]^{-1} M\left(\delta^{*}\right) \pm C B & =0,
\end{aligned}
$$

where again the dependence on $\rho^{*}$ is hidden in the system matrices.

For example, solving (46) for the third-order relay system described in Sec. 2.2 w.r.t. to the bifurcation parameter $\rho$ yields $\rho^{*} \approx 2.099$. This is is the same value at which the sliding bifurcation of a simple orbit has been numerically detected (see Fig. 9). Similar conditions can be obtained for asymmetric orbits as well as for orbits of higher periodicity.

\section{Multisliding Orbits}

We have seen that periodic solutions characterized by sliding sections can be generated via a sliding bifurcation of simple orbits. We show now that these

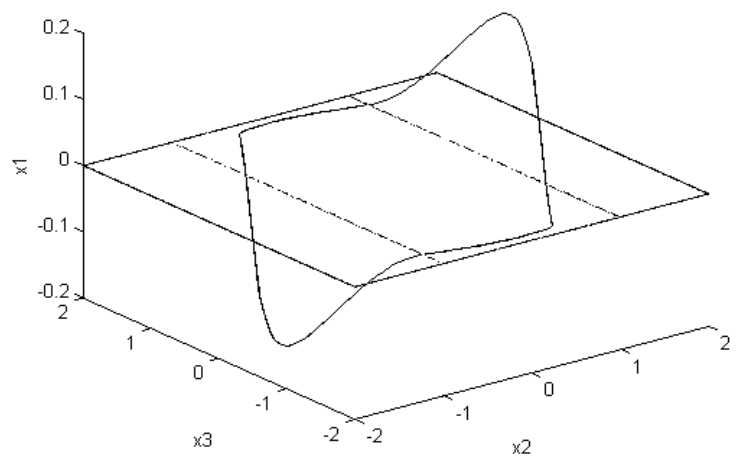

(a)

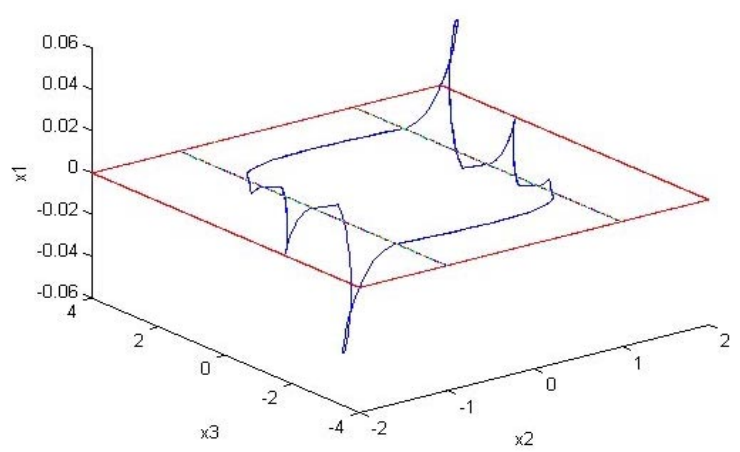

(c) sliding orbits can themselves undergo a class of intricate bifurcations.

Consider, for instance, the third-order relay feedback example with $\lambda=k=-\sigma=\omega=1, \zeta=$ 0.05 and set $\rho=1$. With these parameter values, the system evolves along the sliding orbit exhibited in Fig. 11(a). If we now vary the natural frequency $\omega$ while keeping all the other parameters fixed, we see that, as $\omega$ is increased, the sliding orbit detected for $\omega=1$, bifurcates into a periodic solution characterized by an additional sliding section [see Fig. 11(b)]. We will refer to this type of orbit as a two-sliding periodic solution, i.e. an orbit characterized by two sliding sections per half-period. More generally, we will say that a periodic solution is an $\ell$-sliding orbit when its evolution is characterized by $2 \ell$ sliding sections per period. We will also refer to these novel periodic solutions as "multisliding orbits".

In what follows we show that the transition from a sliding orbit to a multisliding solution described above is due to a novel type of

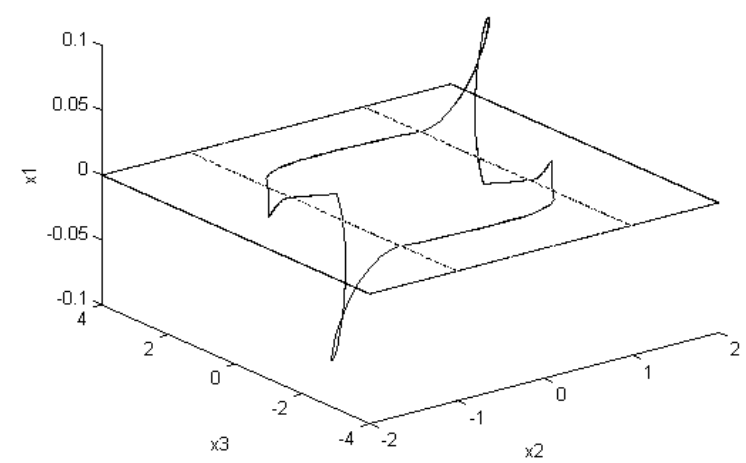

(b)

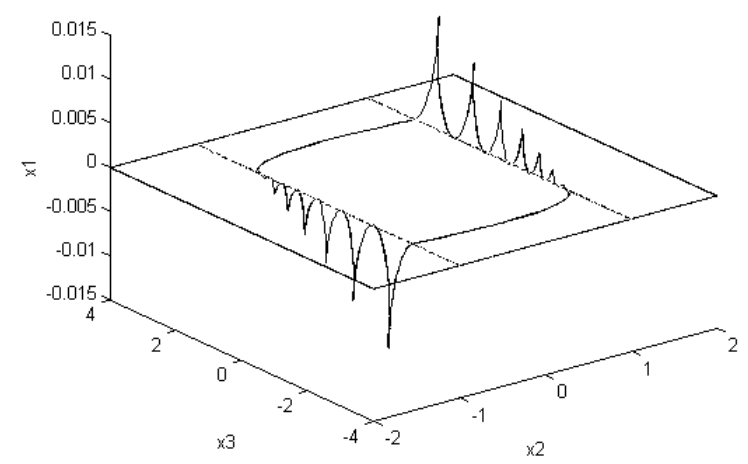

(d)

Fig. 11. State-space diagrams of multisliding orbits for varying values of $\omega$. The cases are (a) $\omega=5$ and $\ell=1$ sliding segment per half-period, (b) $\omega=8$ and $\ell=2$, (c) $\omega=11$ and $\ell=3$, and (d) $\omega=25$ and $\ell=7$. Note how part of the orbit tends to the boundary of the switching hyperplane as $\omega$ increases. 
bifurcation which we shall term "multisliding bifurcation". First, we show how the existence and stability properties of the resulting multisliding orbits can be studied.

\subsection{Existence and local stability}

As for simple and sliding orbits, the existence and stability of multisliding solutions can be studied using an appropriately defined Poincaré map. The multisliding map can be defined as the mapping $\Upsilon: \partial \hat{S} \mapsto \partial \hat{S}$. For a multisliding orbit with $\ell>0$ sliding segments per half period, this is given by

$$
\Upsilon=\left(\Xi^{-}\right)^{\ell} \circ\left(\Xi^{+}\right)^{\ell}=\left(\Sigma \circ \Pi^{-}\right)^{\ell} \circ\left(\Sigma \circ \Pi^{+}\right)^{\ell} .
$$

The sliding map introduced in Sec. 5 corresponds to $\ell=1$. A (possibly asymmetric) one-periodic multisliding orbit defines a fixed point of $\Upsilon$. The algebraic condition is readily derived as in the sliding orbit case reported in Sec. 5. For example, symmetric one-periodic multisliding orbits are found by solving the equation $\left(\Xi^{+}\right)^{\ell}\left(x_{0}\right)=-x_{0}$ constrained by the condition that each iteration of the map $\Xi^{+}$ must fulfill.

The local stability properties of a multisliding orbit can be derived using the Jacobian $D \Upsilon$.

\subsection{Multisliding bifurcation}

Using the third-order model presented in Sec. 2.2 as a representative example, we will now summarise the features of the multisliding bifurcation which describes the transition from a sliding orbit into a multisliding solution. Careful numerical computations indicate that the key to characterize this bifurcation is to look at the behavior of the sliding part of the bifurcating sliding orbit, i.e. the part of the orbit lying within the sliding region. Specifically, we found that a multisliding bifurcation occurs when the sliding part of the bifurcating orbit hits tangentially the boundary of the sliding region.

An example is reported in Fig. 12. Here we see that, as the system parameters are varied, the sliding section of the orbit moves towards the boundary of the sliding strip. When $\omega \approx 7.175$ the orbit then grazes the boundary of the sliding strip $x^{(2)}= \pm 1$ and, for further parameter variations, eventually "escapes" from the sliding region. Thus, past the bifurcation point, we observe the formation of an additional lobe in the nonsliding part of the system solution. Correspondingly the appearance of a further sliding section in the orbit is observed [see Fig. 12(e)] and a two-sliding orbit is so generated.

When the parameter $\omega$ is further varied, this two-sliding solution can be shown to undergo a similar bifurcation, as shown in Fig. 13 thus giving rise to a three-sliding periodic solution. More generally, as shown in Fig. 14, for the system under investigation an entire cascade of multisliding bifurcations can be detected. This "sliding-adding scenario" describes the formation of periodic solutions characterized by an increasing number of sliding sections. Four examples of such multisliding orbits are shown in Fig. 11. Note the geometric twists that the trajectory undergoes outside the switching plane. These are visible as oscillations in the time-domain diagrams plotted in Fig. 15 and are due to the complex conjugate poles of the linear part of the system. Recall that these poles are located in $-\omega \zeta \pm i \omega \sqrt{1-\zeta}$, where $i=\sqrt{-1}$. Because $\zeta=0.05$ is small, the step response of the linear system is quite undamped. Therefore, since a relay switching can locally in time be interpreted as a step response with nonzero initial conditions, the responses observed in Figs. 11-15 contain segments of fast oscillations. Note, also, that the frequency of these fast oscillations increases with $\omega$ and approximately scales as $\omega$.

In the case of the third-order relay feedback system, it is also possible to show that, when a multisliding bifurcation occurs, the sliding section of the bifurcating orbit must graze the boundary of the sliding strip at the point $x^{(1)}=0, x^{(2)}= \pm k, x^{(3)}=$ $\mp b_{2} / b_{1}$. In fact, for the sliding section of the orbit to be tangent to the boundary of the sliding strip we must have $x^{(2)}= \pm 1$ and $\dot{x}^{(2)}=0$. From the reduced-order sliding model (19), we can then derive that:

$$
\dot{x}^{(2)}=-\frac{b_{2}}{b_{1}} x^{(2)}+x^{(3)}=0
$$

and, since $x^{(2)}= \pm 1$, we thus have

$$
x^{(3)}=\mp \frac{b_{2}}{b_{1}}
$$

as expected. Hence, for the parameter values set in Sec. 2.2, we have the tangency $x^{(3)}=\mp 2$. This agrees well with the simulation reported in Figs. 12 and 13 .

Finally, it is worth mentioning here that the sliding-adding scenario depicted in Fig. 14 can yield 


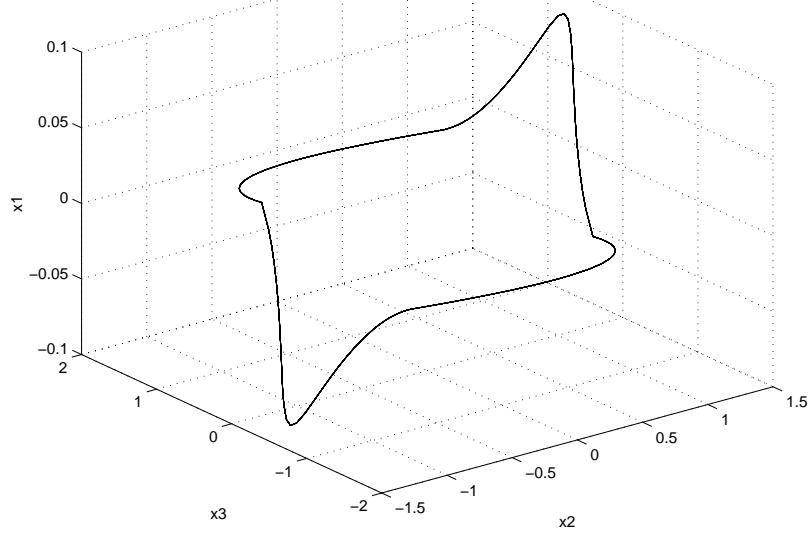

(a)

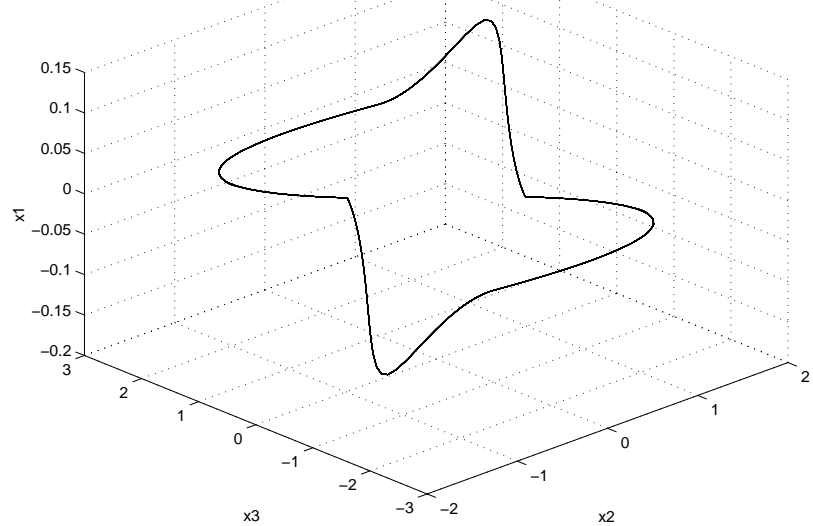

(c)

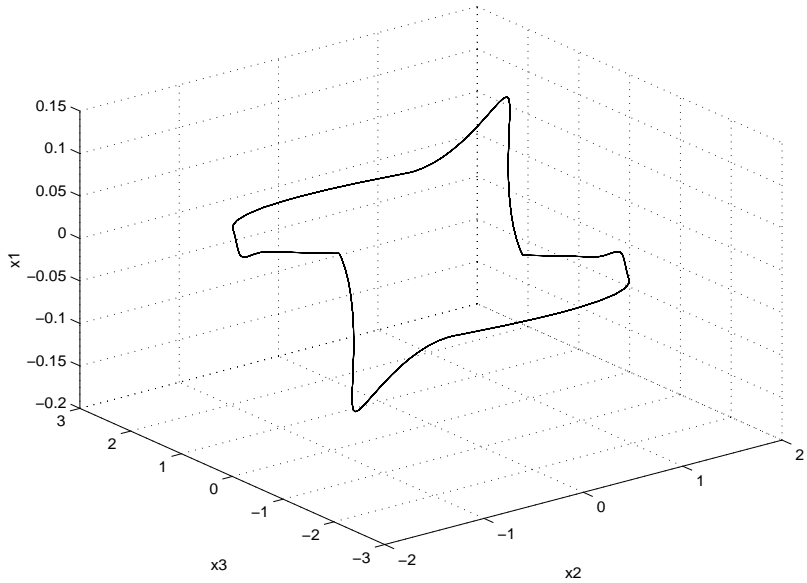

(e)

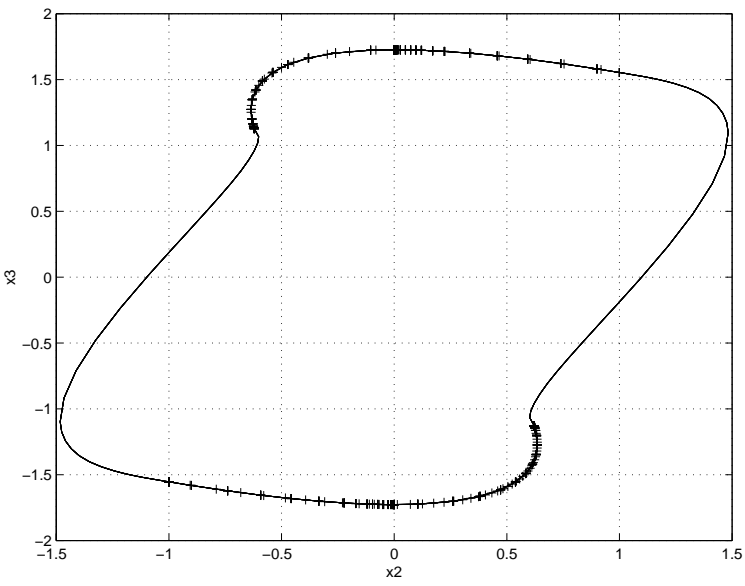

(b)

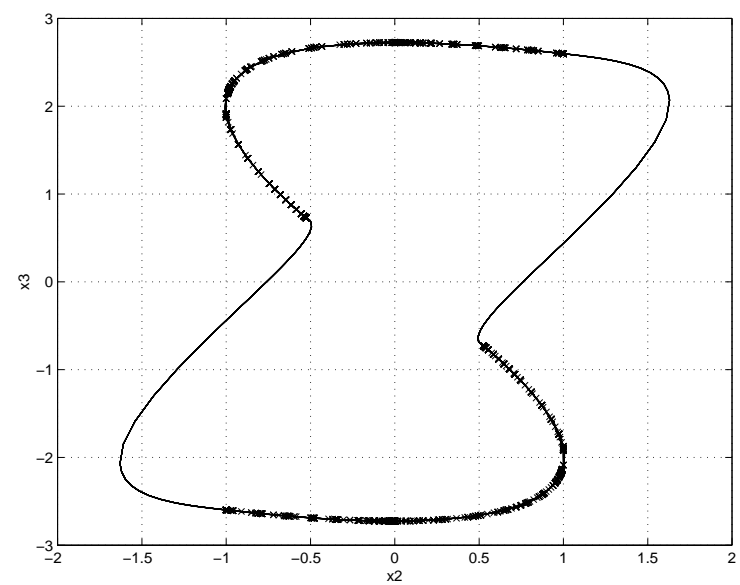

(d)

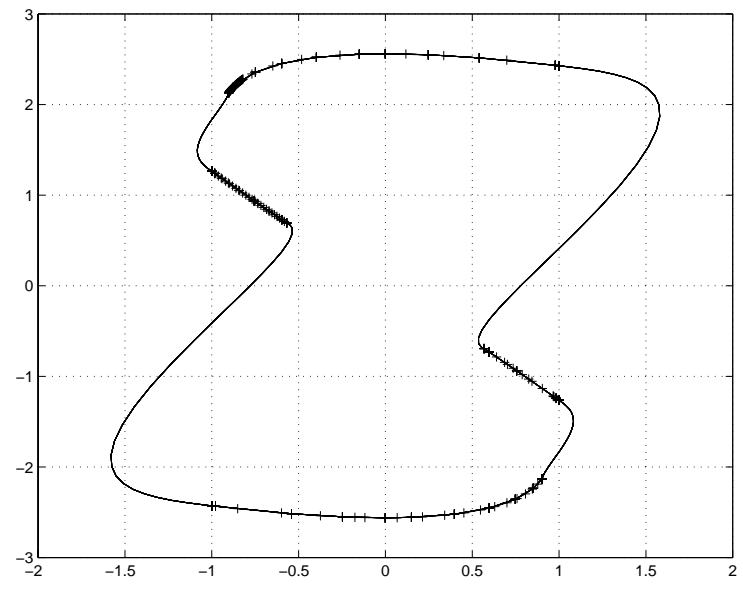

(f)

Fig. 12. Local analysis of the multisliding bifurcation (c) occurring when $\omega=7.1750$, which causes the transition from the sliding orbit observed when $\omega=6.9$ (a) to the two-sliding solution detected for $\omega=7.5$ (e). The projection of the orbits on the switching plane are reported in (b), (d) and (f). Here, the sliding segments of the orbits are depicted by crosses while the periodic connections (which do not belong to the switching hyperplane) are represented by solid line. Note that at the bifurcation point (d), a lobe of the sliding part of the orbit becomes tangent to the boundary of the sliding strip $\left(x_{2}= \pm 1\right)$. 

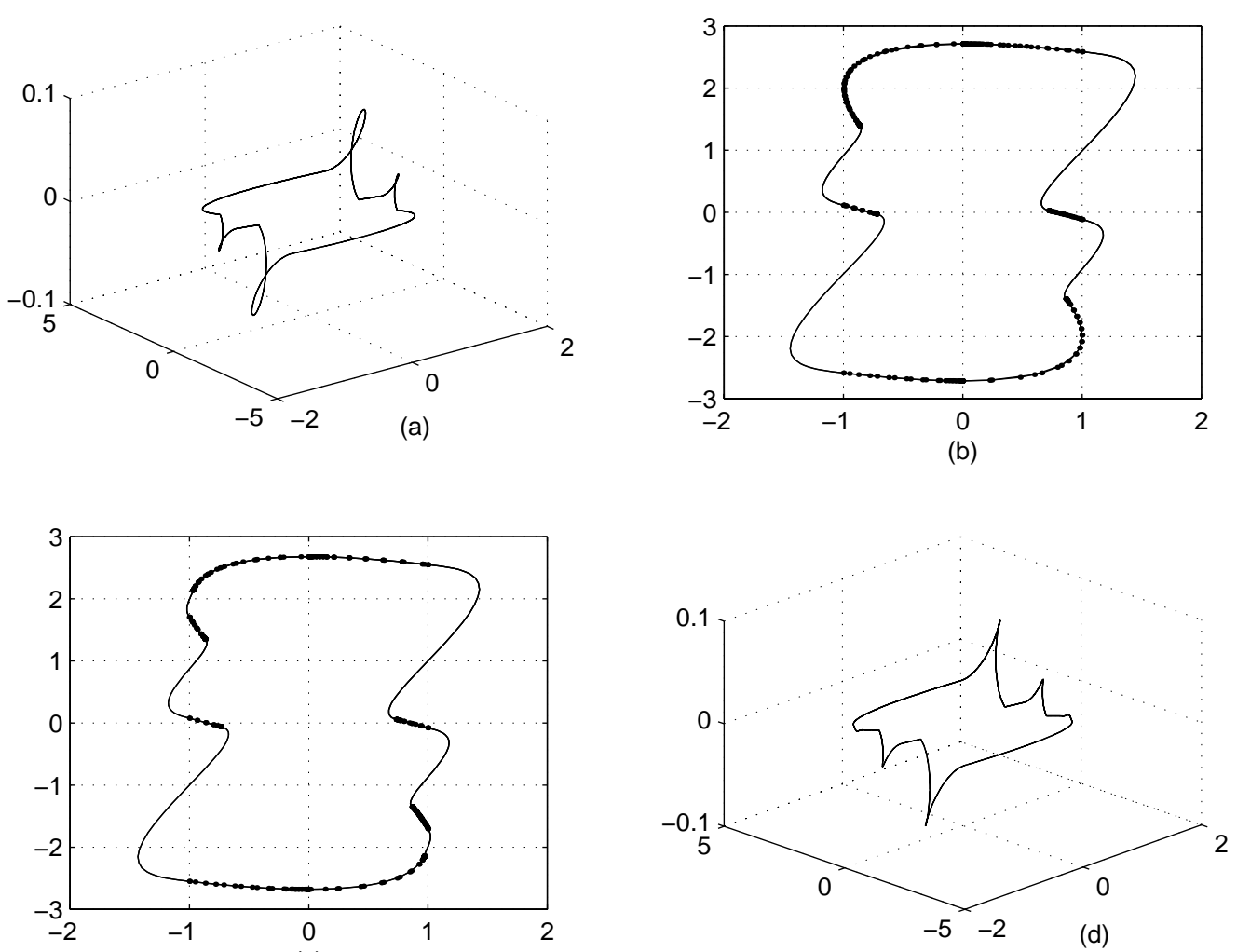

Fig. 13. Multisliding bifurcation observed at $\omega=10.24$, which causes the transition from a two-sliding orbit (a) to a threesliding solution (d). As shown in (b) at the bifurcation point, a lobe of the sliding part of the orbit (highlighted by dots in the figure) is tangent to the boundary of the sliding strip. As the parameter is varied through the bifurcation point then an additional sliding segment occurs (c).

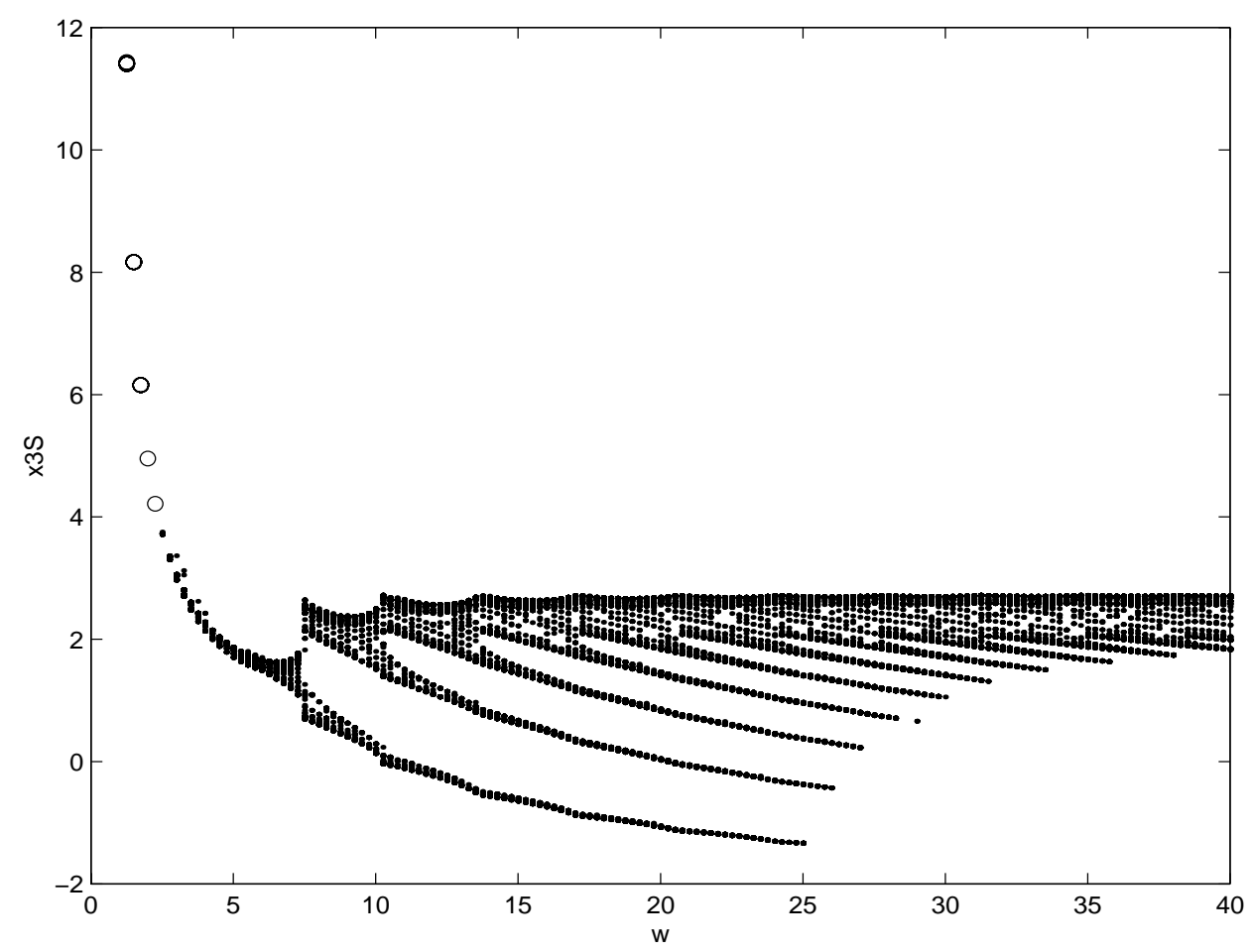

Fig. 14. Multisliding bifurcation diagram. 


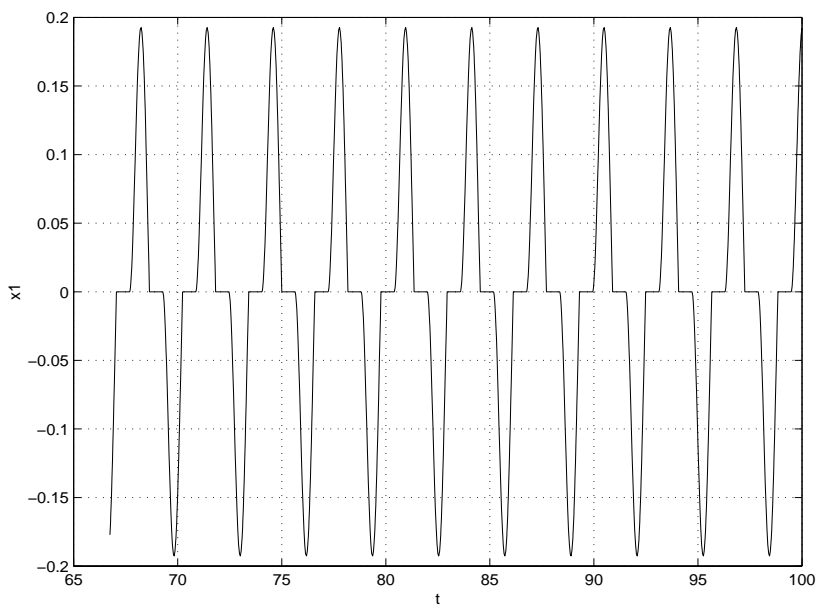

(a)

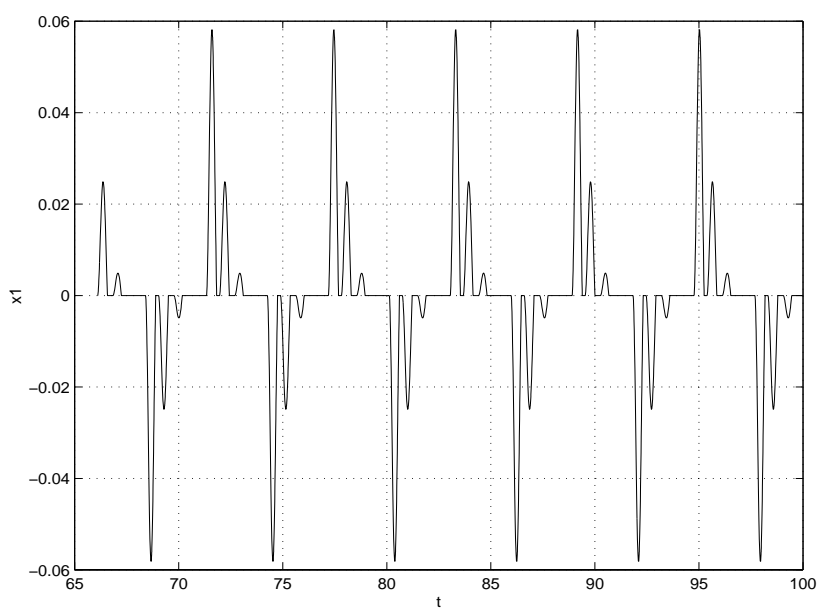

(c)

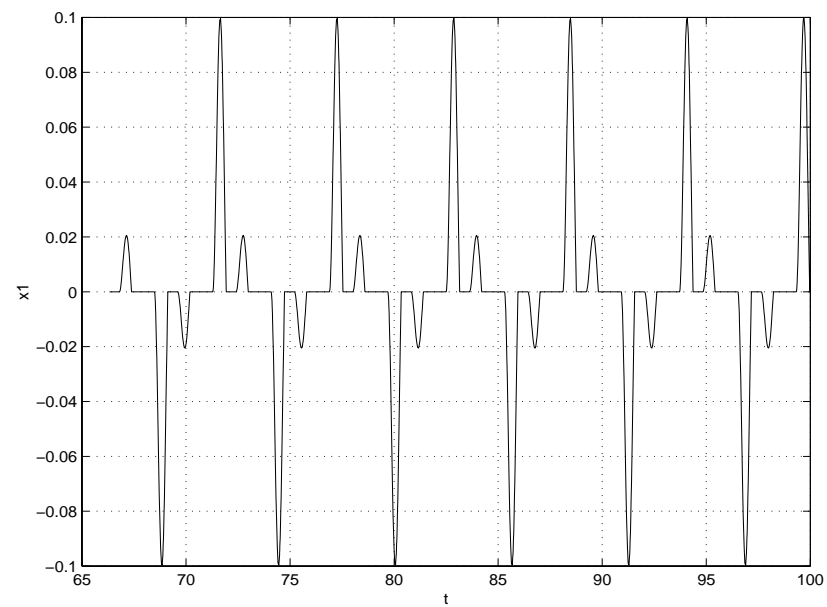

(b)

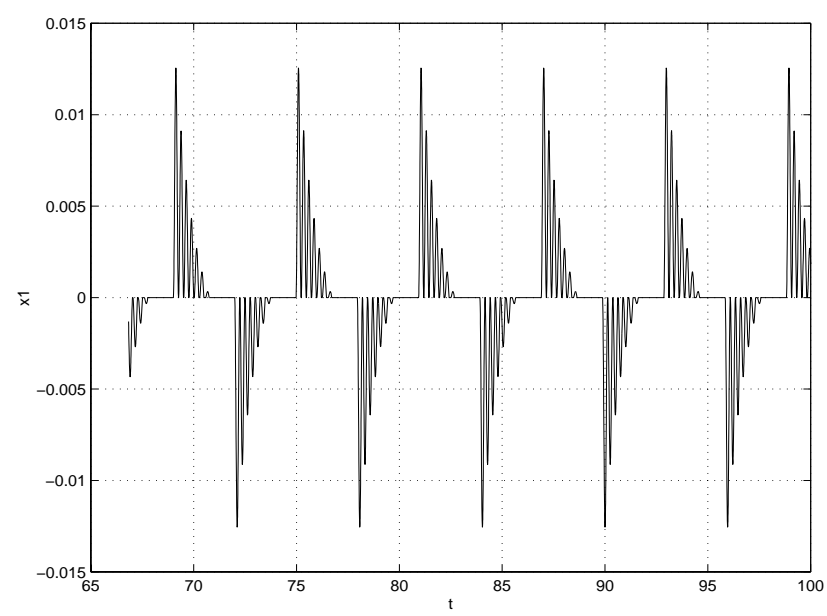

(d)

Fig. 15. Time-domain diagrams of the $x^{(1)}$ component of multisliding orbits for varying values of $\omega$. The cases are (a) $\omega=5$ and $\ell=1$ sliding segment per half-period, (b) $\omega=8$ and $\ell=2$, (c) $\omega=11$ and $\ell=3$, and (d) $\omega=25$ and $\ell=7$. Note how the frequency of the fast oscillations increases with $\omega$.

the formation of multisliding orbits with an arbitrarily large number of sliding segments per period. As the number of sliding segments tends to infinity, the multisliding part of the orbit tends to evolve along the boundary of the sliding strip $\partial \hat{S}$ (corresponding to the lines $x^{(2)}= \pm 1$ for the third-order example under investigation). This can be clearly seen in Fig. 11(d), where a symmetric multisliding orbit with seven sliding segments is shown. In this figure we see that the orbit consists of two different parts together with their symmetric counterparts: a section consisting of shorter and shorter sliding segments which get closer and closer to $\partial \hat{S}$; a long sliding segment taking the system evolution across $\hat{S}$. We conjecture that the limiting solution of the sliding-adding scenario, if it exists, will be characterized by evolving partly along the boundary of the sliding strip $\partial \hat{S}$.

\section{Conclusions}

Piecewise smooth dynamical systems have received considerable attention in the control literature over the last decade. One reason for this is the increasing number of new applications which require the use of control strategies involving both conventional continuous-time controllers together with discrete-event switching. Modeling of such control systems leads often to piecewise smooth model 
dynamics as, for instance, in two recent applications in the automotive industry and flight management, see [Varaiya, 1993] and [Tomlin et al., 1998] respectively.

In this paper, we have discussed the bifurcation behavior of a class of relay feedback systems which are particularly important in the applications mentioned above. Using a set of appropriately defined Poincaré maps we have seen that, contrary to what is usually assumed in the control literature, symmetric, unforced relay feedback systems can indeed exhibit asymmetric self-oscillations. Moreover, we have outlined the existence in these systems of novel classes of local bifurcations, termed sliding and multisliding bifurcations, which cause the formation of orbits characterized by one or more sliding sections.

Multisliding bifurcations have also been shown to organize a peculiar sliding-adding scenario describing the formation of orbits characterized by an increasing number of sliding sections as the system parameters are varied.

During our investigation, we obtained strong preliminary evidence that the bifurcation phenomena discussed in the paper can lead to the occurrence of more complex attractors in relay feedback systems symmetric and without hysteresis (for the case of relay systems with hysteresis see [Cook, 1985]). In Fig. 16, we see for instance a seemingly aperiodic stable solution clearly organized around an asymmetric multisliding orbit. ${ }^{1}$ Current work is directed towards the characterization of this and other similar complex attractors.

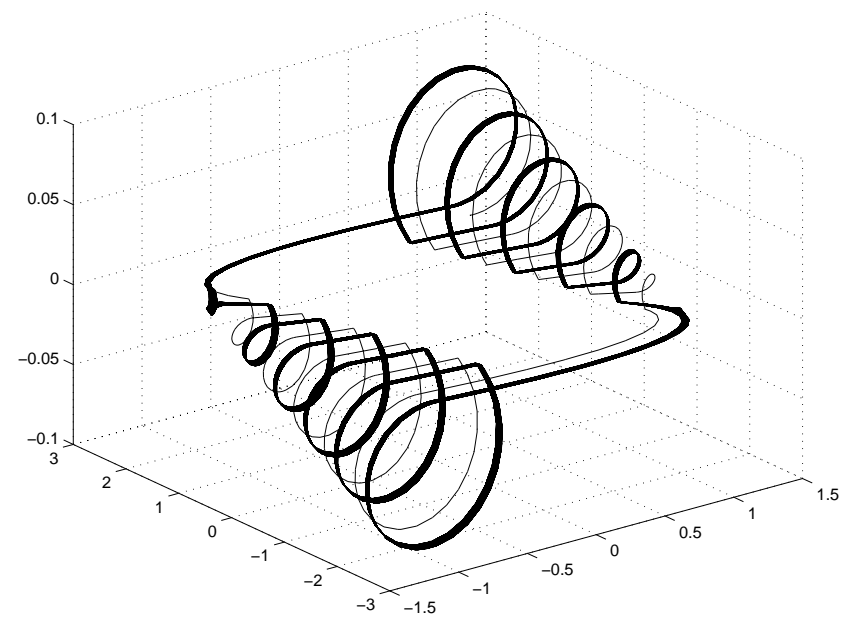

Fig. 16. Seemingly aperiodic attractor observed for $\sigma=-1$, $\rho=1, \omega=10, \zeta=-0.07, \lambda=0.05$. The underlying organizer is clearly an asymmetric multisliding solution.
Finally, we conjecture that sliding and multisliding bifurcations are types of bifurcations to be found in a wider class of piecewise smooth dynamical systems. There is evidence to believe, for example, that the so-called stick-slip oscillations in friction oscillators could be reinterpreted as instances of this more general class of bifurcation events [Dankowicz \& Nordmark, 1999]. Future work will address this generalization.

\section{Acknowledgments}

The authors would like to thank Ms. Francesca Mazzula who developed the numerical code used to compute the simulations reported in the paper during her M.Sc. course at the University of Benevento and the University of Bristol. Also, M. di Bernardo is grateful to Dr. Arne Nordmark, KTH, Stockolm, Sweden for the fruitful discussions on sliding and multisliding bifurcations and Profs. M. I. Feigin and Y. S. Fedosenko, Volga Academy of Water Transport, Nizhniy Novgorod, Russia for their help and advice with some of the Russian literature on the problem. The work by Karl Henrik Johansson was supported by the Swedish Foundation for International Cooperation in Research and Higher Education. M. di Bernardo acknowledges support from the Nuffield Foundation, London (scheme 'NUF-NAL') and the International Centre for Advanced Studies in Nizhniy Novgorod, Russia (INCAS- project no. 99-1-02).

\section{References}

Aizerman, M. A. \& Pyatnitskii, E. S. [1974] "Foundations of a theory of discontinuous systems. I-II," Autom. Remote Contr. 35(7), 1066-1079; 1242-1262.

Amrani, D. \& Atherthon, D. P. [1989] "Designing autonomous relay systems with chaotic motion," Proc. 28th IEEE Conf. Decision and Control, Vol. 1, Tampa, FL, pp. 932-936.

Andronov, A. A., Khaikin, S. E. \& Vitt, A. A. [1965] Theory of Oscillators (Pergamon Press, Oxford).

Åström, K. J. [1995] "Oscillations in systems with relay feedback," in Adaptive Control, Filtering, and Signal Processing, eds. Åström, K. J., Goodwin, G. C. \& Kumar, P. R., IMA Volumes in Mathematics and its Applications, Vol. 74 (Springer-Verlag), pp. 1-25.

Åström, K. J. \& Hägglund, T. [1995] PID Controllers: Theory, Design, and Tuning, 2nd edition, Instrument Society of America, Research Triangle Park, NC.

\footnotetext{
${ }^{1}$ Our preliminary computations show that this orbit is indeed chaotic since it is characterized by a positive Lyapunov exponent.
} 
Atherton, D. P. [1975] Nonlinear Control Engineering: Describing Function Analysis and Design (Van Nostrand Reinhold Co., London, UK).

Brockett, R. W. [1993] "Hybrid models for motion control systems," in Essays in Control: Perspectives in the Theory and Its Applications, eds. Trentelman, $\mathrm{H}$. \& Willems, J. (Birkhäuser, Boston), pp. 29-53.

Cook, P. A. [1985] "Simple feedback systems with chaotic behaviour," Syst. Contr. Lett. 6, 223-227.

Dankowicz, H. \& Nordmark, A. [2001] "On the origin and bifurcations of stick-slip oscillations," Physica D136, 280-307.

di Bernardo, M., Champneys, A. R. \& Budd, C. J. [1998a] "Grazing, skipping and sliding: Analysis of the nonsmooth dynamics of the DC/DC buck converter," Nonlinearity 11, 858-890.

di Bernardo, M., Garofalo, F., Glielmo, L. \& Vasca, F. [1998b] "Switchings, bifurcations and chaos in DC/DC converters," IEEE Trans. Circuits Syst. Part I 45, 133-141.

di Bernardo, M. \& Vasca, F. [1999] "On discrete time maps for the analysis of bifurcations and chaos in DC/DC converters," IEEE Trans. Circuits Syst. Part $I$, accepted for publication.

diBernardo, M., Kowakzyk, P. \& Nordmark, A. "Bifurcations in dynamical systems with sliding: Derivation of normal form maps," in preparation.

Fedosenko, Y. [1976] "On the structure of the phase portrait and periodic solutions of autonomous dynamical systems with impacts," Prikladiaia Matematika $i$ Mechanika (Applied Mathematics and Mechanics) 40(1), 619-629.

Feigin, M. I. [1970] "Doubling of the oscillation period with $c$-bifurcations in piecewise continuous systems," PMM 34, 861-869.

Feigin, M. I. [1974] "On the generation of sets of subharmonic modes in a piecewise continuous system," PMM 38, 810-818.

Filippov, A. F. [1988] Differential Equations with Discontinuous Righthand Sides (Kluwer Academic Publishers).

Flügge-Lotz, I. [1953] Discontinuous Automatic Control (Princeton University Press).

Fridman, L. M. \& Levant, A. [1996] "Higher order sliding modes as a natural phenomenon in control theory," in Robust Control via Variable Structure \& Lyapunov Techniques, eds. Garofalo, F. \& Glielmo, L., Lecture notes in Control and Information Science, Vol. 217 (Springer-Verlag), pp. 107-133.

Genesio, R. \& Tesi, A. [1990] "Chaos prediction in a third order relay system," Internal Report RT 29/90. Dipartimento di Sistemi ed Informatica, University of Florence, Italy.

Gonçalves, J. M., Megretski, A. \& Dahleh, M. A. [1999] "Global stability of relay feedback systems," Technical Report Preprint LIDS-P-2458, Dept. of EECS, MIT, Cambridge, MA.
Hawkins, J. T. [1887] "Automatic regulators for heating apparatus," Trans. Am. Soc. Mech. Engin. 9, p. 432.

Holzhueter, T. \& Klinker, T. [1998] "Control of a chaotic relay system using the OGY method," Zeitschrift fuer Naturforschung Section A53(12), 1029-1036.

Johansson, K. H., Barabanov, A. \& Åström, K. J. [1997] "Limit cycles with chattering in relay feedback systems," Proc. 36th IEEE Conf. Decision and Control, San Diego, CA, 1997.

Johansson, K. H., Rantzer, A. \& Åström, K. J. [1999] "Fast switches in relay feedback systems," Automatica 35(4), 539-552.

Kuznetsov, Y. [1995] Elements of Applied Bifurcation Theory (Springer-Verlag).

Megretski, A. [1996] "Global stability of oscillations induced by a relay feedback," in Preprints IFAC 13th World Congress, Vol. E, San Francisco, CA, pp. 49-54.

Morse, A. S. [1995] "Control using logic-based switching," in Trends in Control. A European Perspective, ed. Isidori A. (Springer), pp. 69-113.

Neimark, Y. L. [1972] Methods of Pointwise Mappings in the Theory of Nonlinear Oscillations (Nauka, Moscow) (in Russian).

Nordmark, A. B. [1991] "Non-periodic motion caused by grazing incidence in impact oscillators," J. Sound Vib. 2, 279-297.

Norsworthy, S. R., Schreier, R. \& Temes, G. C. [1997] Delta-Sigma Data Converters - Theory, Design, and Simulation (IEEE Press, NY).

Nusse, L. E \& Yorke, J. A. [1994] "Border collision bifurcation: An explanation for observed bifurcation phenomena," Phys. Rev. E49, 1073-1076.

Popp, K. \& Shelter, P. [1990] "Stick-slip vibrations and chaos," Philos. Trans. R. Soc. A332(1624), 89-105.

Swift, J. \& Wiesenfeld, K. [1984] "Suppression of period doubling in symmetric systems," Phys. Rev. Lett. 52(9), 705-708.

Tomlin, C., Pappas, G. J. \& Sastry, S. [1998] "Conflict resolution for air traffic management: A study in multi-agent hybrid systems," IEEE Trans. Autom. Contr. 4, 504-521.

Tsypkin, Ya. Z. [1984] Relay Control Systems (Cambridge University Press, Cambridge, UK).

Utkin, V. I. [1992] Sliding Modes in Control Optimization (Springer-Verlag, Berlin).

Varaiya, P. [1993] "Smart cars on smart roads: Problems of control," IEEE Trans. Autom. Contr. 38(2), 195-207.

\section{Appendix}

The following result gives a class of relay feedback systems that do not have asymmetric periodic solutions. 
Proposition 1. The relay feedback system (1)-(3) with

$$
\begin{aligned}
& C(s I-A)^{-1} B=K \prod_{k=1}^{n}\left(s+\lambda_{k}\right)^{-1}, \\
& K>0, \quad \lambda_{1}>\cdots>\lambda_{n}>0, \quad n>0
\end{aligned}
$$

has no asymmetric one-periodic orbits.

Proof. Note first that for $n=1$ and $n=2$ the relay feedback system is globally stable, so there exist no periodic solutions at all. Therefore, assume that $n>2$. Moreover, assume by contradiction that there exists an asymmetric one-periodic orbit with switching times $h_{1} \neq h_{2}$. It follows from

$$
\begin{aligned}
C x_{1}= & -C\left[I-e^{A\left(t_{1}+t_{2}\right)}\right]^{-1}\left[e^{A\left(t_{1}+t_{2}\right)}\right. \\
& \left.-2 e^{A t_{2}}+I\right] A^{-1} B \\
C x_{2}= & C\left[I-e^{A\left(t_{1}+t_{2}\right)}\right]^{-1}\left[e^{A\left(t_{1}+t_{2}\right)}\right. \\
& \left.-2 e^{A t_{1}}+I\right] A^{-1} B,
\end{aligned}
$$

that $C x_{1}\left(t_{1}, t_{2}\right)=0$ and $C x_{2}\left(t_{1}, t_{2}\right)=0$ for $\left(t_{1}, t_{2}\right)=\left(h_{1}, h_{2}\right)$. Note that if $\left(h_{1}, h_{2}\right)$ is a solution, then $\left(h_{2}, h_{1}\right)$ is also a solution. It is no restriction to assume that $A=\operatorname{diag}\left(-\lambda_{1}, \ldots,-\lambda_{n}\right)$. The solution of (A.1) satisfies

$$
C\left(I-e^{\tilde{A}}\right)^{-1}\left(e^{\tilde{A}}-2 e^{\tilde{A} t_{2} /\left(t_{1}+t_{2}\right)}+I\right) \tilde{A}^{-1} \tilde{B}=0,
$$

where $\tilde{A}=A\left(h_{1}+h_{2}\right)$ and $\tilde{B}=B\left(h_{1}+h_{2}\right)$. Note that

$$
\begin{gathered}
C(s I-\tilde{A})^{-1} \tilde{B}=\tilde{K} \prod_{k=1}^{n}\left(s+\tilde{\lambda}_{k}\right)^{-1}, \\
\tilde{K}>0, \quad \tilde{\lambda}_{1}>\cdots>\tilde{\lambda}_{n}>0
\end{gathered}
$$

has the same form as the original system. We next show that there exists no diagonal $\hat{A}, \hat{B}$ and $\hat{C}$ with

$$
\begin{gathered}
\hat{C}(s I-\hat{A})^{-1} \hat{B}=\hat{K} \prod_{k=1}^{n}\left(s+\hat{\lambda}_{k}\right)^{-1}, \\
\hat{K}>0, \quad \hat{\lambda}_{1}>\cdots>\hat{\lambda}_{n}>0,
\end{gathered}
$$

such that the function

$$
f(t)=\hat{C}\left(I-e^{\hat{A}}\right)^{-1}\left(e^{\hat{A}}-2 e^{\hat{A} t}+I\right) \hat{A}^{-1} \hat{B},
$$

has $\operatorname{roots} \tau_{1}, \tau_{2}>0$ with $\tau_{1} \neq \tau_{2}$. It then follows that there exist no two solutions $\left(h_{1}, h_{2}\right)$ and $\left(h_{2}, h_{1}\right)$ with $h_{1} \neq h_{2}$ for (47) and (48), which proves the result. and

Note that $f(0)=-\hat{C} \hat{A}^{-1} \hat{B}=\hat{K} \prod_{k=1}^{n} \hat{\lambda}_{k}^{-1}>0$

$$
f^{\prime}(t)=-2 \hat{C}\left(I-e^{\hat{A}}\right)^{-1} e^{\hat{A} t} \hat{B} .
$$

From the stability of $\hat{A}$ and $f(0)>0$, it follows that $f^{\prime}(t)<0$ for all $t>0$. Hence, the equation $f(t)=0$ only has one solution and the proof is complete. 